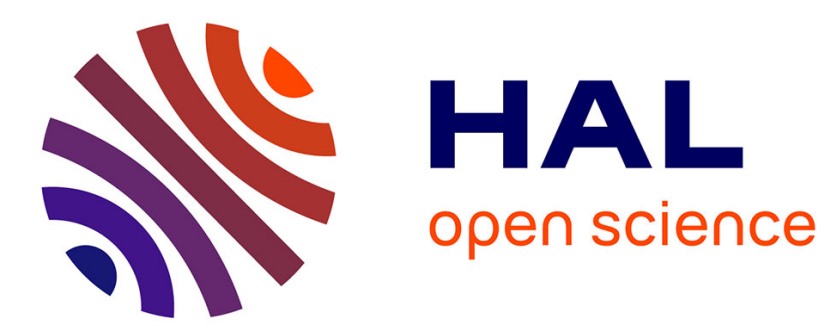

\title{
Multiple droplets formation in ultrathin bridges of rigid rod dispersions
}

\author{
Andrey V Subbotin, Alexander Semenov
}

\section{To cite this version:}

Andrey V Subbotin, Alexander Semenov. Multiple droplets formation in ultrathin bridges of rigid rod dispersions. Journal of Rheology, 2020, 64 (1), pp.13-27. 10.1122/1.5115464 . hal-02905256

\section{HAL Id: hal-02905256 \\ https://hal.science/hal-02905256}

Submitted on 23 Jul 2020

HAL is a multi-disciplinary open access archive for the deposit and dissemination of scientific research documents, whether they are published or not. The documents may come from teaching and research institutions in France or abroad, or from public or private research centers.
L'archive ouverte pluridisciplinaire HAL, est destinée au dépôt et à la diffusion de documents scientifiques de niveau recherche, publiés ou non, émanant des établissements d'enseignement et de recherche français ou étrangers, des laboratoires publics ou privés. 


\section{Multiple droplets formation in ultrathin bridges of rigid rod dispersions}

Andrey V. Subbotin, and Alexander N. Semenov

Citation: Journal of Rheology 64, 13 (2020); doi: 10.1122/1.5115464

View online: https://doi.org/10.1122/1.5115464

View Table of Contents: https://sor.scitation.org/toc/jor/64/1

Published by the The Society of Rheology

\section{ARTICLES YOU MAY BE INTERESTED IN}

Rotational motions of repulsive graphene oxide domains in aqueous dispersion during slow shear flow Journal of Rheology 64, 29 (2020); https://doi.org/10.1122/1.5120323

Capillary breakup extensional electrorheometry (CaBEER) Journal of Rheology 64, 43 (2020); https://doi.org/10.1122/1.5116718

Relaxation time of dilute polymer solutions: A microfluidic approach Journal of Rheology 61, 327 (2017); https://doi.org/10.1122/1.4975933

A review of thixotropy and its rheological modeling Journal of Rheology 63, 477 (2019); https://doi.org/10.1122/1.5055031

Rheological quantification of the extent of dissolution of ultrahigh molecular weight polyethylene in meltcompounded blends with high density polyethylene

Journal of Rheology 64, 1 (2020); https://doi.org/10.1122/1.5113705

Capillary breakup extensional magnetorheometry Journal of Rheology 64, 55 (2020); https://doi.org/10.1122/1.5115460

\section{Master your flow}

with the MCR Rheometer series
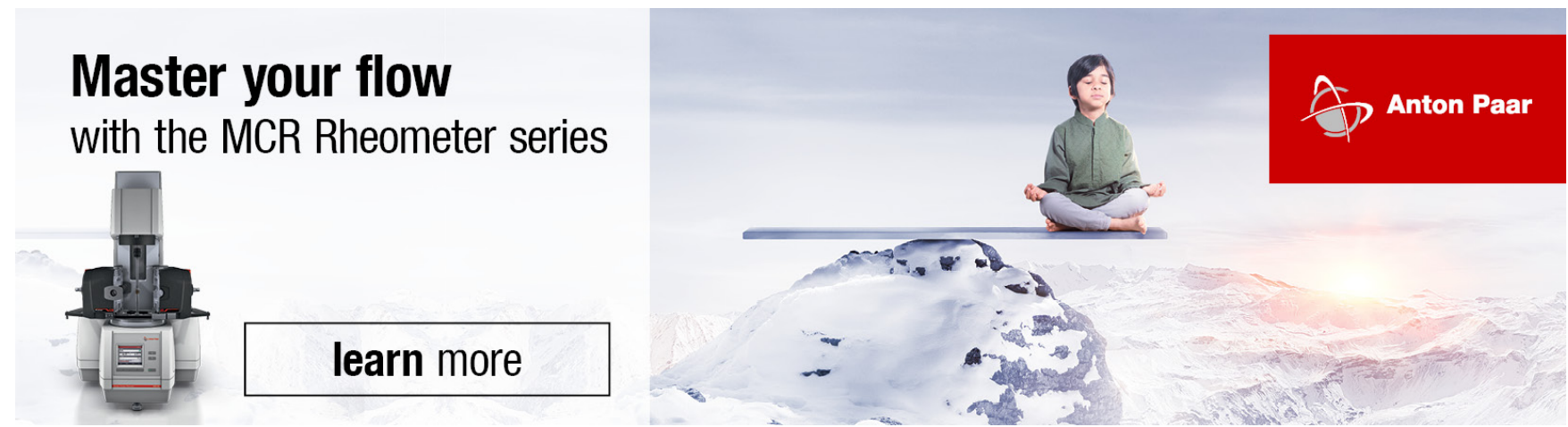


\title{
Multiple droplets formation in ultrathin bridges of rigid rod dispersions
}

\author{
Andrey V. Subbotin ${ }^{1,2}$ and Alexander N. Semenov ${ }^{3}$ \\ ${ }^{1}$ A.V. Topchiev Institute of Petrochemical Synthesis, Russian Academy of Sciences, Leninskii prosp. 29, \\ Moscow 119991, Russia \\ ${ }^{2}$ A.N. Frumkin Institute of Physical Chemistry and Electrochemistry, Russian Academy of Sciences, \\ Leninskii prosp. 31, Moscow 119071, Russia \\ ${ }^{3}$ Institut Charles Sadron, CNRS-UPR 22, Universite de Strasbourg, 23 rue du Loess, BP 84047, \\ 67034 Strasbourg Cedex 2, France
}

(Received 18 June 2019; final revision received 21 October 2019; published 13 November 2019)

\begin{abstract}
In the present paper, we study the rheology of the dispersion of rigid rods in the course of capillary thinning of a liquid bridge formed during extension of a liquid droplet between two solid surfaces. Emergence of multiple annular microdroplets of solvent on the surface of the bridge is predicted. This process comes into play once the radius of the jet decreases below a critical value smaller than the rod length. Formation of the solvent annuli initially occurs by an activation mechanism, whereby the rods become trapped inside the jet core. The kinetics of the annular droplets growth in the regimes of both high and low activation barriers are considered in detail. Several kinetic regimes are studied, including activation, the linear regime when the annular droplets grow exponentially, and the consecutive nonlinear regime of significant core thinning and slow coalescence and growth of droplets. The phase-separation mechanism proposed here for dispersions of rigid rods in the ultrathin jet regime (when jet thickness $a$ is much smaller than the rod length $L$ ) effectively replaces the classical Plateau-Rayleigh pinching; the proposed mechanism can, therefore, prevent the bridge from breaking in this regime, thereby opening up a new route for fiber formation in these systems. (C) 2019 The Society of Rheology. https://doi.org/10.1122/1.5115464
\end{abstract}

\section{INTRODUCTION}

The pinching-off dynamics of liquid jets is extensively studied and attracts wide scientific interest by virtue of different technological applications including inkjet and 3D printing [1-4], spraying [3,5], and fiber formation [6-8]. The most significant progress has been achieved in the studies of capillary thinning of Newtonian jets [9-14]. Based on the analysis of the hydrodynamic equations, several regimes of jet breakup have been identified [15]. The inertial regime is realized at low Ohnesorge numbers, $O h=\eta / \sqrt{\rho \gamma a} \ll 1$, i.e., when the jet is thick enough (here $a$ is the radius of the bridge, $\rho$ is the density of the liquid, $\gamma$ is its surface tension, and $\eta$ is its viscosity). The Reynolds number is large in this regime, $\operatorname{Re}=\rho v_{z} L_{z} / \eta \gg 1$ (here $L_{z}$ is the characteristic length of the pinching bridge and $v_{z}$ is the characteristic axial velocity), and the characteristic breakup time is $\tau_{I} \sim \sqrt{\rho a^{3} / \gamma}$ [16]. (Typically, $L_{z} \sim a$ and $v_{z} \sim a / \tau$ in the inertial regime, so $\operatorname{Re} \sim 1 / O h \gg 1$.) Another-viscousregime is emerged for $O h \gg 1$, that is, for highly viscous liquids or when the jet is relatively thin. Inertial effects become negligible in this regime: $\operatorname{Re} \ll 1$, so the breakup time is $\tau_{V} \sim \eta a / \gamma$ with $L_{z} \gg a[17,18]$. However, both regimes fail close to the breakup point, so a new viscoinertial regime is emerged wherein both the inertia and the viscosity are equally important, while the local Reynolds number is $\operatorname{Re} \sim 1$ [19]. The diagram of regimes in coordinates $\left(O h_{0}, a_{0}\right)$ and transitions between these regimes have been studied recently $[15,20]$ (here $a_{0}$ is the initial jet thickness and $\left.O h_{0}=\eta / \sqrt{\rho \gamma a_{0}}\right)$. Noteworthily, an addition of surfactants affects the pinch-off of liquid threads and results in the occurrence of multiple microthreads [21]. An analysis of the pinchoff dynamics of complex non-Newtonian liquids has also been done recently [22-24].

The breakup dynamics is particularly challenging in the case of jets formed by polymer liquids and other complex liquids such as dispersions of particles [25]. One relevant application domain of polymer liquids is spinning of fibers, which is well known in nature and is widely involved in technological processes like artificial fiber production and electrospinning [6-8,26,27]. Experiments reveal that polymer liquid jets show much more complex behavior than Newtonian jets $[6,28]$. The threads formed by solutions of flexible polymers can show exponential thinning, formation of periodic beads-on-a-string structures [28-33] and blistering patterns [34-38] attributed, in particular, to migration of polymer molecules in the thinner regions due to the stressconcentration coupling effect [39-42]. An exponential thinning law was established in the regime when polymer coils start to stretch, leading to the strain hardening effect $[28,29]$. The effect of viscoelasticity in the beads-on-a-string structures formation has been studied based on the Oldroyd-B and FENE-P constitutive models [43-47]. They show formation of structures involving spherical beads separated by narrow threads with stretched chains and constant polymer concentration. The blistering patterns result from instability mechanisms that differ from the classical Plateau-Rayleigh (PR) pinching instability. A generalized description involving the constitutive equation, the Navier-Stokes equations, and the equation for polymer concentration has been proposed to 
elucidate the blistering effect arisen at the final stage of pinching [39]. A different approach elucidating the blistering effect was proposed recently [48-50] based on a molecular model. Within this theory, the blistering comes from the flow-induced phase separation under extension. More precisely, it was predicted that a polymer/solvent demixing in unentangled polymer solutions can arise due to flow-induced orientation of polymer chains, leading to a subsequent reversal of their effective interactions from repulsive to attractive. As a result the elongated chains tend to microseparate and form a network of fibrils tending to compress laterally by squeezing the solvent out to the jet surface $[49,50]$.

The jets formed by solutions of stiff polymers such as polypeptides, DNA, cellulose, aromatic polyamide copolymers, etc., as well as dispersions of rodlike particles (F-actin, microtubes, carbon nanotubes, etc.) are even less studied. Yet, such systems are very interesting as they play an important role in living organisms and can show liquid crystalline phases with high degrees of order which exhibit both viscoelasticity and orientational elasticity [51-55]. Synthetic stiffchain polymers (Kevlar, Vectra, etc.) are also capable of forming high performance fibers [56-58].

In this paper, we focus on capillary thinning of solutions of rigid rods in the regime of ultrathin jet when its diameter is smaller than the rod length and the rods are highly oriented along the jet axis. Stable orientation of rigid rod suspensions has been demonstrated in a strong extensional flow [59]. Such a regime can arise at the terminal stage of capillary thinning of a jet stretched with an electric force in an electrospinning process $[8,27,60-62]$ or in a liquid bridge formed by stretching of a liquid droplet $[4,7,63-65]$. We show that the solvent/rods demixing can be triggered by the capillary forces activating and driving the drainage of the solvent to the jet surface, while leaving the rods inside the jet core. Multiple solvent annular droplets are formed around the jet as a result.

In Subsection II A, we describe the model solution of rodlike polymers and its main parameters including the dynamical time scales and length scales. Subsection II B is devoted to the thermodynamics of the rodlike polymer solution, in particular, to the osmotic pressure, which plays an important role for the dynamical instability effect we consider. In Subsection II C, we briefly consider orientational dynamics of the rods in a prescribed extensional flow. Finally, in Subsection II D, we outline the jet thinning setup, the solution/flow regime we focus on, and the main effect (capillary-induced phase separation) considered in the paper. The scope of Secs. III-VII is also announced there. The main conditions specifying the system we consider are listed in Sec. VII.

\section{THE MODEL AND THE FLOW REGIME}

\section{A. The main parameters of the model solution of rodlike polymers}

Let us consider a semidilute solution (dispersion) of rigid rodlike macromolecules of length $L$ and diameter $d, d \ll L$. The average concentration of rods is $c=N / V$ where $N$ is the total number of rods in the volume $V$. Here, we focus on solution in the semidilute concentration regime where, on the one hand, the rods are crowded, $c L^{3} \gg 1$, but, on the other hand, the volume fraction of rods, $\phi=\pi L d^{2} c / 4$, is below the nematic transition point: $\phi \ll \phi_{n} \sim 4 d / L$, so that the solution is isotropic at equilibrium. The rotational dynamics of rods in such a crowded system is characterized by the diffusion constant $D_{r} \simeq\left(a_{t} / L\right)^{2} D_{r 0}$, where $a_{t}$ is the characteristic diameter of the "tube" created by the neighboring rods and $D_{r 0}$ is the rotational diffusion constant of an isolated rod in the dilute solution without entanglements [66]. Note that $D_{r 0}=6 / \tau_{r}$, where $\tau_{r}$ is the characteristic time necessary for a rod to move on a distance $\sim L$ along its axis, $\tau_{r} \simeq \zeta_{\|} L^{3} / T$ [66]. Here, $T=k_{B} T_{\text {abs }}$ is the temperature in energy units $\left(k_{B}\right.$ is the Boltzmann constant), $\zeta_{\|}=2 \pi \eta_{s} / k_{H}$ is the parallel component of the friction coefficient per unit rod length, $\eta_{s}$ is the viscosity of the solvent, and $k_{H}$ is the hydrodynamic factor, which for semidilute solutions reads $k_{H} \simeq \ln \left(\xi_{H} / d\right) \simeq 0.5 \ln (1 / \phi)$, where $\xi_{H} \sim \xi \equiv(c L)^{-1 / 2}$ is the relevant screening length $[49,66]$. This length is roughly the mean distance from an arbitrary point to the nearest rod. The tube diameter for an isotropic solution of rods is estimated as $a_{t} \simeq L \sqrt{\beta} /\left(c L^{3}\right)$, where $\beta \sim 1000$ is a numerical factor $[66,67]$. Thus, the rotation of rods gets hindered if $c L^{3}>\sqrt{\beta}$ : the relevant time scale is $1 / D_{r}, 1 / D_{r} \gg \tau_{r}$.

\section{B. Thermodynamics of uniform bulk solution}

In this part, we discuss the thermodynamic properties of a solution of rodlike polymers, including osmotic pressure which is important for the flow dynamics considered in Secs. III-VI. The relevant thermodynamic potential $F$ of a solution of rodlike polymers can be written as (per unit volume) [68]

$$
F(\mu, T, c)=\Omega(\mu, T)+F_{\text {rod }}(\mu, T, c),
$$

where $\Omega(\mu, T)$ is the grand thermodynamic potential of the pure solvent per unit volume and

$$
F_{\text {rod }}(\mu, T, c) \simeq T c \ln (c / e)+\psi(\mu, T) c+F_{\mathrm{int}}(c, T, \mu)
$$

is the free energy density of the rods $(e \approx 2.718)$. In the above formulas, $\mu$ is the chemical potential of the solvent and $\psi(\mu, T)$ is solvation energy per rod. The first term in Eq. (2) is due to translational entropy of rods, and $F_{\text {int }}$ is the free energy density due to their interactions; it can be represented as a virial expansion: $F_{\text {int }}=(T / 2) B(\mu, T) c^{2}+\cdots$, where $B$ is the second virial coefficient (excluded volume). The total thermodynamic potential is $\mathcal{F}=V F$, and the total pressure in the system reads

$$
P_{\mathrm{tot}}=-\frac{\partial \mathcal{F}}{\partial V} \simeq-\Omega(\mu, T)+c T+c^{2} \frac{\partial\left(F_{\mathrm{int}} / c\right)}{\partial c} .
$$

The last equation generally is written as

$$
P_{\text {tot }}=P+\Pi,
$$


where $P=-\Omega(\mu, T)$ is the solvent pressure and

$$
\Pi=c^{2} \frac{\partial\left(F_{\mathrm{rod}} / c\right)}{\partial c}=c T+c^{2} \frac{\partial\left(F_{\mathrm{int}} / c\right)}{\partial c}
$$

is the osmotic pressure of rods. The interaction term $F_{\text {int }}$ can be neglected for sufficiently dilute solutions, namely, well below the concentration of nematic transition, $c \ll 4 / L^{2} d$ [52]. For highly oriented rods, the latter condition can be weakened as $c d^{2} L \ll 1$. In this regime, the osmotic pressure is determined by the ideal-gas term: $\Pi \simeq c T$. Note that $P$ is equal to the local pressure in the regions free of rods; the mean concentration of solvent molecules in these regions is equal to $c_{s, \text { loc }}=\partial P / \partial \mu=-\partial \Omega(\mu, T) / \partial \mu$. Thus, $c_{s, \text { loc }}$ depends only on $\mu$, but not on $c$. On the other hand, the total number of solvent molecules is $N_{s}=-\partial \mathcal{F} / \partial \mu \simeq V c_{s, \text { loc }}-N \partial \psi / \partial \mu$. Thus, the total volume

$$
V=N_{s} v_{s, \text { loc }}+N v_{r}
$$

where $v_{s, \text { loc }}=1 / c_{s, \text { loc }}$ is the local volume per solvent molecule (away from the rods) and $v_{r}=(\partial \psi / \partial \mu) v_{s, \text { loc }}$. The first term on the rhs of Eq. (5) is the intrinsic volume of all solvent molecules (at a given $\mu, T$ ), while the second term is the effective additional volume due to solute particles. The volume per particle, therefore, is $v_{r}=\partial \psi /\left.\partial P\right|_{T}$; for bulky rods of thickness $d$ much exceeding the size of solvent molecule the volume $v_{r}$ is defined sterically, $v_{r} \simeq \pi L d^{2} / 4$.

In the general case, the osmotic pressure $\Pi=c T+\Pi_{\text {int }}$, where the interactional part $\Pi_{\text {int }}$ is defined as the last term in Eq. (4). Here and below, we assume that the rods interact by steric repulsion only (no attraction), so (cf. [49,51])

$$
\Pi \approx c T\left[1+\left(\frac{L}{d} I+4\right) \frac{\phi}{1-\phi}\right],
$$

where factor $I=I(\alpha)$ is defined in Eq. (7) with orientational distribution function, Eq. (10); the term " 4 " in brackets corresponds to the excluded volume of two strictly parallel rods which is equal to $8 v_{r}$.

\section{Orientational effects for rods under a prescribed extensional flow}

In the presence of a uniaxial extensional flow (along the axis z) characterized by the tensor of velocity gradients $\dot{\boldsymbol{\varepsilon}} \equiv \nabla \mathbf{v}=\dot{\varepsilon} / 2\left(3 \mathbf{e}_{z} \mathbf{e}_{z}-\mathbf{I}\right)$, where $\mathbf{v}=\mathbf{v}(\mathbf{r})$ is the fluid velocity, $\nabla$ is the gradient operator, and $\mathbf{I}$ is a unit tensor, the rods get oriented. [The corresponding velocity field in cylindrical coordinates $(z, r, \varphi)$ is given by $\mathbf{v}=\dot{\varepsilon} z \mathbf{e}_{z}-(\dot{\varepsilon} r / 2) \mathbf{e}_{r}$, where $\mathbf{e}_{z}$ is the unit vector along the $z$-axis and $\mathbf{e}_{r}$ is radial unit vector.] The rod orientations can be described by the distribution density function $f(\mathbf{n}), \int f(\mathbf{n}) \mathrm{d} \mathbf{n}=1$, where the unit vector $\mathbf{n}$ is directed along the rod axis and $d \mathbf{n}$ is the surface element on the unit sphere $|\mathbf{n}|=1$ around the direction $\mathbf{n}$ so that $c f(\mathbf{n}) \mathrm{d} \mathbf{n}$ is the number of rods (in a unit volume) oriented inside the solid angle dn around $\mathbf{n}$. The effective rotational diffusion coefficient for the rods in the anisotropic state is given by $\bar{D}_{r} \simeq D_{r} I^{-2}$ with the orientational factor [66]

$$
I=\frac{4}{\pi} \int f\left(\mathbf{n}_{1}\right) f\left(\mathbf{n}_{2}\right)\left|\mathbf{n}_{1} \times \mathbf{n}_{2}\right| \mathrm{d} \mathbf{n}_{1} \mathrm{~d} \mathbf{n}_{2} .
$$

The free energy density of the oriented rods [cf. Eq. (2)] is now augmented with an additional orientational contribution

$$
\begin{aligned}
\Delta F_{\text {rod }}(\mu, T, c) \simeq & c T \int f(\mathbf{n}) \ln (4 \pi f(\mathbf{n})) \mathrm{d} \mathbf{n} \\
& +c \int f(\mathbf{n}) U(\mathbf{n}) \mathrm{d} \mathbf{n} .
\end{aligned}
$$

The first term in Eq. (8) is the orientational entropy and the potential $U(\mathbf{n})$ in the second term is the effective energy of a rod with orientation $\mathbf{n}$ due to the hydrodynamic flow. This energy is equal to the work against the friction torque to achieve a given orientation. It can be found from the torque balance equation [66]

$$
\mathbf{n} \times \nabla_{\mathbf{n}} U(\mathbf{n})=-\zeta_{r} \mathbf{n} \times \dot{\boldsymbol{\varepsilon}} \cdot \mathbf{n} .
$$

Here, the rotational friction coefficient $\zeta_{r}=T / \bar{D}_{r}$ and $\nabla_{\mathbf{n}}$ is the gradient in the orientational space. Minimization of $\Delta F_{\text {rod }}$ with respect to $f(\mathbf{n})$ after using (8) yields

$$
\begin{aligned}
f(\mathbf{n}) & =\text { const. } \cdot \exp \left(\frac{3 \dot{\varepsilon}}{4 \bar{D}_{r}} \cos ^{2} \theta\right) \\
& =\text { const. } \cdot \exp \left(\alpha \cos ^{2} \theta\right),
\end{aligned}
$$

where $\cos \theta=\mathbf{n e}_{z}$ and const. is found from the normalization condition. Note that the free energy, Eq. (8), does not change the osmotic pressure of the solution. The parameter $\alpha=3 \dot{\varepsilon} / 4 \bar{D}_{r}$ is obtained from Eq. (10) self-consistently. For highly oriented rods with $\alpha \gg 1$, the orientational factor $I \sim 1 / \sqrt{\alpha}$, and one gets $\alpha \simeq \dot{\varepsilon} \tau_{r} / 8$ for $\dot{\varepsilon} \tau_{r} \gg\left(L / a_{t}\right)^{2}$. In this case, the characteristic value of the angle between the rod axis and $z$-axis is smaller than the magnitude of angular fluctuations inside the tube

$$
\langle\theta\rangle \simeq 1 / \sqrt{\alpha}<\left(a_{t} / L\right) \sim \sqrt{\beta} /\left(c L^{3}\right) .
$$

The rotational diffusion of such oriented rods is not hindered by entanglements any more. Below, we focus on the regime of highly oriented rods, $\alpha \gg 1$, which is true if $\dot{\varepsilon} \tau_{r} \gg 1$.

\section{Capillary thinning of the solution bridge and instability effects}

Now, we consider a droplet of the solution confined between two parallel plates and stretched by moving the plates, thereby forming a liquid bridge [Fig. 1(a)]. Once the bridge is formed, the plate motion is stopped, but the bridge thickness keeps decreasing due to capillary effects. We, therefore, consider the regime where the extension rate $\dot{\varepsilon}$ is defined by the bridge surface tension $\gamma$ and its radius $a$ : $\dot{\varepsilon} \simeq \gamma /(3 \eta a)[37,69]$, where $\eta$ is the effective viscosity of the 
(a)

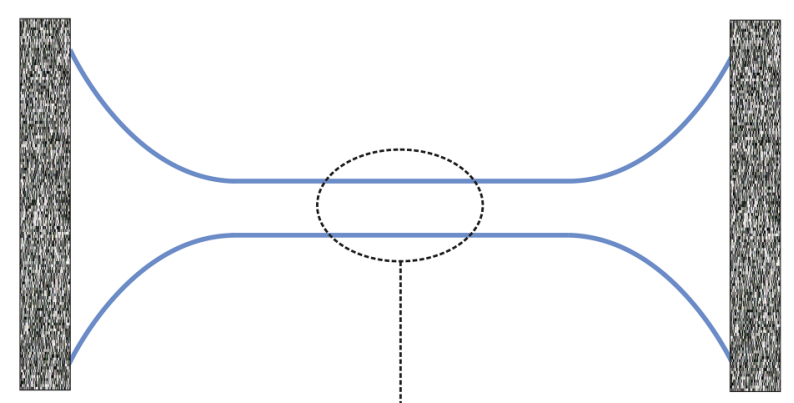

(b)
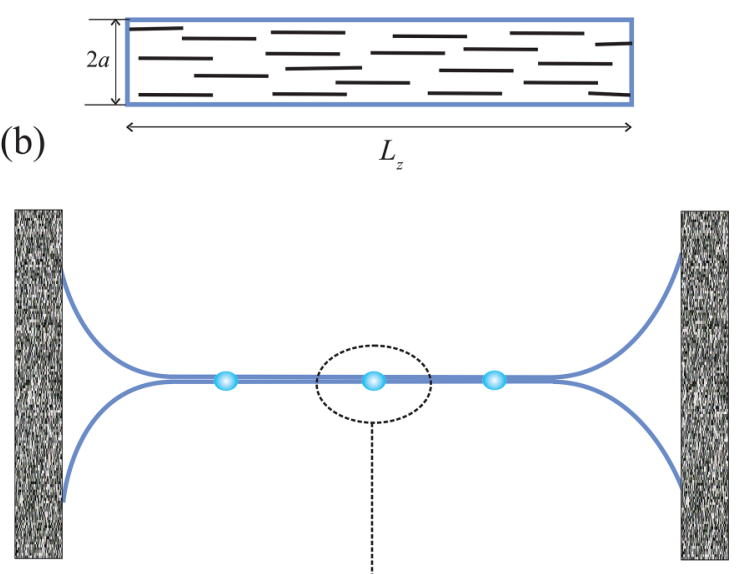

(c)

(d)

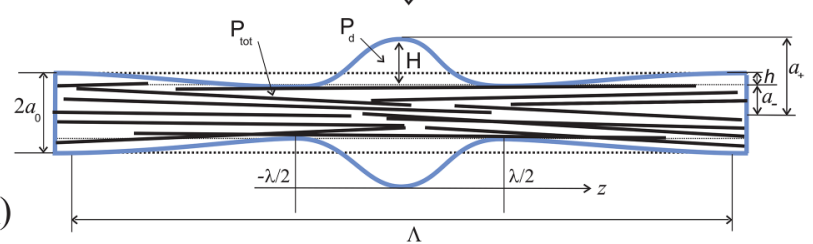

FIG. 1. Illustration of the liquid bridge (a) and cylindrical jet (b) formed as a result of extension of a millimeter-size droplet of solution placed between 2 solid surfaces in the regime when the radius of the jet $a>L / 2$; (c) the bridge coated with annular droplets in the regime $a_{0} \ll L / 2$; (d) longitudinal cross section of the core/droplet structure. Here, $H=a_{+}-a_{-}$is the annular droplet height, $\lambda$ is its length along the axis, $V_{d}$ is its volume, $a_{-}$is the radius of the droplet/core interface, $h=a_{0}-a_{-}$is the maximum shrinkage depth, $\Lambda \gg a_{0}$ is the total length of the core segment affected by the droplet (i.e., where the core radius $\left.a_{\text {core }}(z)<a_{0}\right) . P_{\text {tot }}$ is the total pressure inside the jet core, and $P_{d}$ is the solvent pressure in the droplet.

solution. Therefore, the condition of highly oriented rods, $\dot{\varepsilon} \tau_{r} \gg 1$, is satisfied if

$$
\frac{\gamma}{\eta a} \gg \frac{T}{L^{3} \eta_{s}}
$$

Below, we focus on the central part of the bridge [see Fig 1(b)], which can be approximated as a long, thin and nearly uniform cylindrical jet of length $L_{z}$ and radius $a \ll L_{z}$ in the regime, where inertial effects can be neglected. The latter condition is satisfied if $a a^{*} \gg L_{z}^{2}$, where $a^{*}=\eta^{2} / \rho \gamma$. Note that the last inequality is compatible with $a \ll L_{z}$ and that the two above conditions imply that $a \ll a^{*}$. The latter condition is not necessarily very strong. For example, with the typical values of $\rho \sim 1 \mathrm{~g} \mathrm{~cm}^{-3}$ and $\gamma \sim 100 \mathrm{erg} \mathrm{cm}^{-2}$, one gets $a^{*}>1 \mathrm{~cm}$ if the elongational viscosity of the solution $\eta>10 \mathrm{P}$. In this case, the general restriction on the jet length reads $L_{z} \ll 1 \mathrm{~cm}$, which can be easily satisfied experimentally. Another requirement is that the jet radius $a$ does not change much on the length-scale $L$ (rod length): $L|\partial a / \partial z| \ll a$ (here, $z$ is the elongation axis), which is equivalent to $L \ll L_{z}$.

It is well known that a cylindrical liquid jet of the radius $a<a^{*}$ is unstable with respect to small undulations and should breakup in droplets during the time $\tau_{\mathrm{PR}}=6 \eta a / \gamma$ in accordance with the PR mechanism [1-7]. In the case of highly oriented rods in an extentional flow, the effective viscosity (equal to $1 / 3$ of the conventional elongational viscosity) is given by

$$
\eta \simeq \eta_{s}\left(1+\frac{\pi}{18 k_{H}} c L^{3}\right) .
$$

This equation comes from the Doi-Edwards theory [66]. It shows the cubic dependence of elongational viscosity on the rod length just like for flexible polymer chains in a highly stretched state [37]. Therefore, the breakup time is $\tau_{P R}=(6 \eta a / \gamma) \sim\left(\eta_{s} a L^{2} / \gamma \xi^{2}\right) \quad\left(c L^{3}=L^{2} / \xi^{2} \gg 1\right)$. The condition $\tau_{\mathrm{PR}} D_{r 0} \ll 1$ ensures that the rods are kept highly oriented by the flow during the jet thinning process. The latter condition is equivalent to condition (12) and can be reduced to $L d^{2} /(\phi a) \gg T / \gamma$. It can be easily verified that this condition is always strongly satisfied (for any polymer volume fraction $\phi$ ) with typical values of parameters $L, d, a, T, \gamma$ stated below Eq. (22) [and below Eq. (64)].

The classical PR mechanism assumes that the radius of the jet strongly exceeds the molecular size (the rod length in the present case). It is shown below, however, that the flow dynamics is drastically different when the jet radius becomes smaller than the rod length $L$. In this case, the dynamics of short-wave fluctuations with the period smaller than $L$ becomes qualitatively different from that for long-wave fluctuations with a period exceeding the rod length. In the longwave fluctuation regime, the solution can be considered as a continuum medium and its pinch-off dynamics is described by the PR mechanism with the characteristic time $\tau_{\mathrm{PR}}$ quoted in the previous paragraph. By contrast, in the present paper, we demonstrate that a new instability mechanism sets in at shorter length scales, which is much faster, with the characteristic time scale much shorter than $\tau_{\mathrm{PR}}$. This instability results in emergence of solvent annuli outside the jet core [Fig. 1(c)]. In Sec. III, we study the formation dynamics of a single annular droplet and calculate the relevant activation barrier (always focusing on the regime, when the average number of rods in the cross section of the thread is large: $n=\pi a^{2} c L \gg 1$ ). In Secs. IV and V, we consider formation of multiple annular droplets, while the late stages of the droplets evolution including their coalescence are studied in Sec. VI.

\section{CAPILLARY-INDUCED SHORT-LENGTH INSTABILITY}

Let us consider a cylindrical jet of radius $a_{0} \ll L$ and concentration $c=c_{0}$ (in the semidilute regime $c_{0} L^{3} \gg 1$, 
$\left.\phi_{0} \ll 1\right)$, which is formed upon stretching of a rodlike polymer solution droplet. The length of the jet is assumed to be large, $L_{z} \gg L$, with many rods in the cross section of the thread, $n=\pi a^{2} c L \gg 1$. We note that generally the solvent is allowed to squeeze out and form tiny annular droplets around the jet due to thermal fluctuations. Such a process would lead to the core/droplet structure with all the rods located in the core of radius $\simeq a_{0}$ and the solvent both inside and outside the core, as illustrated in Figs. 1(c) and 1(d). The total pressure $P_{\text {tot }}$ inside the jet core is a sum of the solvent pressure $P$ and the osmotic pressure $\Pi_{0}$ of rods at $c=c_{0}: P_{\text {tot }}=P+\Pi_{0}$ [cf. Eq (3a)]. At equilibrium, the solvent pressure in the droplet, $P_{d}$ [cf. Fig. 1(d)], must be equal to the local solvent pressure in the core, $P$. For an infinitesimal annular droplet $[H \rightarrow 0$, cf. Fig. 1(d)], the pressure $P_{d}$ is defined by the capillary pressure $P_{\text {cap }}=\gamma C \simeq \gamma / a_{0}$ (here $\gamma$ is the surface tension and $C$ is the total local curvature of the annulus surface, i.e., the sum of curvatures in two perpendicular directions) and the atmospheric pressure $P_{a}$ outside: $P_{d}=P_{\text {cap }}+P_{a}$.

It is important that the osmotic pressure of rods is much weaker than the capillary pressure, $\Pi_{0} \ll \gamma / a_{0}$ for any $\phi_{0} \ll 1$. In fact, even for $\phi_{0} \sim 0.5$ (the worst case), the osmotic pressure $\Pi_{0} \sim 4 c_{0} T \sim T / d^{2} L$ [cf. Eq. (6)], so $\Pi_{0} a_{0} / \gamma \sim\left(T / \gamma d^{2}\right) a_{0} / L . \quad$ With $\quad a_{0} \ll L, \quad d \sim 2 \mathrm{~nm}$, $\gamma \sim 100 \mathrm{dyn} / \mathrm{cm}, T_{\mathrm{abs}} \approx 300 \mathrm{~K}$, we get $p \equiv \Pi_{0} a_{0} / \gamma \ll 10^{-2}$. The small "osmotic" parameter $p$ is central for the perturbation theory presented below.

Obviously, the droplet will grow spontaneously if $P>P_{d}$, that is, $P-P_{a}>P_{\text {cap }}$. On the other hand, an equilibrium at the jet core/air interface implies that the total pressure in the core, $P_{\text {tot }}=P+\Pi_{0}$, must be equal to the sum of the capillary pressure $P_{\text {cap }}$ and atmospheric pressure, $P_{\text {tot }}=P_{\text {cap }}+P_{a}$. Hence, we get

$$
P-P_{a}=P_{\text {cap }}-\Pi_{0}
$$

Therefrom, we find that for very small droplet $(H \rightarrow 0)$ $P=P_{d}-\Pi_{0}$. As $\Pi_{0}>0$, the inequality $P>P_{d}$ is not valid, so the surface droplet cannot emerge spontaneously: its formation requires activation. Below, we calculate the corresponding potential energy barrier. We set $P_{a}=0$ for simplicity in what follows.

To calculate the activation energy, let us consider a small but finite deformation of the cylindrical jet so that it adopts a slightly perturbed axially symmetric shape with radius $a=a(z)$ weakly deviating from the initial radius $a_{0}$ : $\left|a-a_{0}\right| \ll a_{0}$ [cf. Fig. 1(d)]. The annular droplet is located in the region $-\lambda / 2 \leq z \leq \lambda / 2$ ( $\lambda$ is the droplet length, $\left.a_{0}<\lambda \ll L\right)$. The rods are confined inside the core of radius $a_{\text {core }}(z), a_{\text {core }}(z)=a(z)$ outside the droplet region, $|z|>\lambda / 2$, and $a_{\text {core }}(z)=a_{-}$for $|z| \leq \lambda / 2$, cf. Fig. 1(d).

The optimum shape of the droplet is found by minimization of its excess surface area $A_{d}=2 \pi \int_{-\lambda / 2}^{\lambda / 2} a(z) \sqrt{1+a_{z}^{\prime 2}} \mathrm{~d} z-$ $2 \pi \lambda a_{-}$(here $a_{z}^{\prime}=\mathrm{d} a / \mathrm{d} z$ ) at the fixed core radius $a_{-}$and droplet volume. It corresponds to a surface of constant curvature $C$ [70], defined by the differential equation

$$
a_{+} a_{-}+a^{2}=\frac{2 a}{C \sqrt{1+a_{z}^{\prime 2}}}, \quad a_{+}+a_{-}=2 / C,
$$

where $a=a(z)$ and $a_{+}$defines the height (thickness) $H$ of the solvent droplet: $H=a_{+}-a_{-}$[cf. Fig. 1(d)]. Thus, the curvature is $C=\left(1 / a_{-}\right)\left(1+\left(H / 2 a_{-}\right)\right)^{-1}$. Here, we assume for simplicity that the radius $a_{-}$of the core/droplet interface is constant; this assumption is justified by the condition $\lambda \ll L$. The solution of Eq. (15) is presented below in the implicit form in terms of the inverse function $z=z(a)$, which has two branches (in both cases $a_{-} \leq a \leq a_{+}$)

$z(a)=\left\{\begin{array}{lr}-z^{*}+\int_{a_{-}}^{a} \mathrm{~d} a \frac{a_{+} a_{-}+a^{2}}{\sqrt{\left(a_{+}^{2}-a^{2}\right)\left(a^{2}-a_{-}^{2}\right)}}, & -z^{*} \leq z \leq 0, \\ \int_{a}^{a_{+}} \mathrm{d} a \frac{a_{+} a_{-}+a^{2}}{\sqrt{\left(a_{+}^{2}-a^{2}\right)\left(a^{2}-a_{-}^{2}\right)}}, & 0 \leq z \leq z^{*} .\end{array}\right.$

The droplet profile for $H=0.5 a_{-}$is shown in Fig. 2.

The length of the annular droplet is found from Eq. (16) using a new variable $\varphi$, which is defined through the formula $a^{2}=\left[\left(a_{+}^{2}+a_{-}^{2}\right)+\left(a_{+}^{2}-a_{-}^{2}\right) \sin \varphi\right] / 2$,

$$
\lambda=\frac{2}{C} \int_{-\pi / 2}^{\pi / 2} \mathrm{~d} \varphi \frac{1+\left(1-a_{-} C\right) \sin \varphi}{\sqrt{a_{-}^{2} C^{2}+2\left(1-a_{-} C\right)(1+\sin \varphi)}}
$$

Similarly, we get the surface area and the volume of the droplet

$$
A_{d}=\frac{4 \pi}{C^{2}} \int_{-\pi / 2}^{\pi 2} \mathrm{~d} \varphi \sqrt{a_{-}^{2} C^{2}+2\left(1-a_{-} C\right)(1+\sin \varphi)}
$$

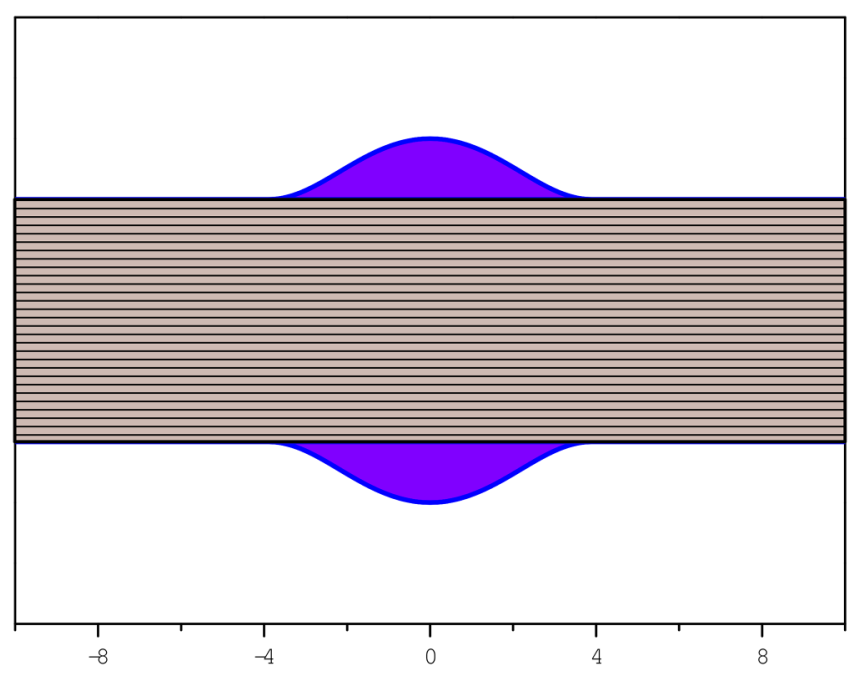

FIG. 2. Annular droplet profile for the jet of core radius $a_{-}=1$ and $H=0.5$. 


$$
\begin{aligned}
V_{d}= & \pi \int_{-\lambda / 2}^{\lambda / 2}\left(a(z)^{2}-a_{-}^{2}\right) \mathrm{d} z \\
= & \frac{2 \pi}{C^{3}} \int_{-\pi / 2}^{\pi / 2} \mathrm{~d} \varphi\left(1+\left(1-a_{-} C\right) \sin \varphi\right) \\
& \times \sqrt{a_{-}^{2} C^{2}+2\left(1-a_{-} C\right)(1+\sin \varphi)}-\pi a_{-}^{2} \lambda
\end{aligned}
$$

For small droplets, $H \ll a_{0}$, Eqs. (17a)-(17c) can be written as expansions for small $H / a_{0}$. The total curvature of the droplet can be represented as $C=\left(1 / a_{-}\right)+\Delta C$, where the increment $\Delta C \simeq-\left(H / 2 a_{0}^{2}\right)$. Moreover, we can set $a_{-} \simeq a_{0}$ since the difference $h=a_{0}-a_{-}$is very small: in fact, $h$ can be found from the volume conservation equation $V_{d} \simeq 2 \pi^{2} a_{0}^{2} H \sim 2 \pi a_{0} h \Lambda$ with $\Lambda \gg a_{0}$ [cf. Fig. 1(d)], leading to $h \sim H a_{0} / \Lambda \ll H$ so that the corrections accounting for nonzero $h / a_{0}$ are subdominant with respect to the terms of the expansion in $H / a_{0}$ that are kept in the equation

$$
\begin{aligned}
\lambda & \simeq 2 \pi a_{0}\left(1-\frac{H}{2 a_{0}}+\frac{3 H^{2}}{16 a_{0}^{2}}\right), \\
A_{d} & \simeq 4 \pi^{2} a_{0}^{2}\left(1-\frac{H}{a_{0}}+\frac{13 H^{2}}{16 a_{0}^{2}}\right), \\
V_{d} & \simeq 2 \pi^{2} a_{0}^{2} H\left(1+\frac{3 H}{2 a_{0}}\right) .
\end{aligned}
$$

Taking into account Eq. (18) and noting that $\mid\left(1 / a_{-}\right)-$ $\left(1 / a_{0}\right)|\ll| \Delta C \mid$ since $h \ll H$, we get

$$
C \simeq \frac{1}{a_{0}}-\frac{V_{d}}{\left(2 \pi a_{0}^{2}\right)^{2}}
$$

The free energy changes as the solvent squeezes from the jet core to the surface and forms a droplet of volume $V_{d}$. The total free energy change (minimal work required to form the droplet) is represented as a sum of osmotic and capillary terms $\Delta \mathcal{F} \simeq \Delta \mathcal{F}_{\Pi}+\Delta \mathcal{F}_{\text {surf }}$. The osmotic increment can be found from $\mathrm{d}\left(\Delta \mathcal{F}_{\Pi}\right)=\Pi \mathrm{d} V_{d}$. During the process, the local concentration of rods in the jet core changes only slightly, $c-c_{0} \sim c_{0} h / a_{0} \ll c_{0}$ since $h \ll H \ll a_{0}$ [see Eq. (23a)], so the osmotic pressure is nearly constant, $\Pi \simeq \Pi_{0}$ [see also justification below Eq. (23a)], and $\Delta \mathcal{F}_{\Pi} \simeq \Pi_{0} V_{d}$. The surface energy $\Delta \mathcal{F}_{\text {surf }}$ increases with $V_{d}$ as $\mathrm{d}\left(\Delta \mathcal{F}_{\text {surf }}\right)=\gamma \Delta C \cdot \mathrm{d} V_{d}$, where $\Delta C$ is defined below Eq. (17c). After integration one gets $\Delta \mathcal{F}_{\text {surf }} \simeq-\pi^{2} \gamma H^{2} / 2$ (recall that $V_{d} \ll a_{0}^{3}, H \ll a_{0}$ ), therefore,

$$
\Delta \mathcal{F} \simeq \Pi_{0} V_{d}-\frac{\gamma V_{d}^{2}}{2\left(2 \pi a_{0}^{2}\right)^{2}}
$$

The energy $\Delta \mathcal{F}\left(V_{d}\right)$ shows a maximum at

$$
V_{d}=V_{d}^{*}=\left(2 \pi a_{0}^{2}\right)^{2} \Pi_{0} / \gamma, \quad H^{*}=V_{d}^{*} /\left(2 \pi^{2} a_{0}^{2}\right)=2 a_{0}^{2} \Pi_{0} / \gamma,
$$

which corresponds to the activation energy

$$
F_{\text {barr }} \simeq 2 \pi^{2} a_{0}^{4} \Pi_{0}^{2} / \gamma
$$

(the corresponding critical height is $H^{*}$ ). For example, using the following typical or assumed values: $T_{\mathrm{abs}} \approx 300 \mathrm{~K}$, $\xi \sim 10 \mathrm{~nm}, L \sim 1 \mu \mathrm{m}, a_{0} \sim 100 \mathrm{~nm}$, and $\gamma \sim 100 \mathrm{erg} \mathrm{cm}^{-2}$, we find $V_{d}^{*} / a_{0}^{3}=(2 \pi)^{2} \Pi_{0} a_{0} / \gamma \sim 10^{-3}$, hence $V_{d}^{*} \ll a_{0}^{3}$ is satisfied. Note that the conditions

$$
H^{*} \ll a_{0}, \quad h \ll a_{0}, \quad\left|c-c_{0}\right| / c_{0} \sim h / a_{0} \ll 1
$$

simply follow from $p \equiv \Pi_{0} a_{0} / \gamma \ll 1$ since $H^{*} / a_{0} \sim p$ and $h / a_{0} \sim H / \Lambda$. The change of the osmotic pressure due to droplet formation can be estimated as

$$
\left|\Pi-\Pi_{0}\right| \sim \Pi_{0}\left|c-c_{0}\right| / c_{0} \sim \Pi_{0} h / a_{0} \sim \Pi_{0} p a_{0} / \Lambda
$$

It is negligibly small for two reasons $(p \ll 1$ and $\left.a_{0} / \Lambda \ll 1\right)$ justifying the approximation $\Pi \simeq \Pi_{0}$ adopted here and below. In fact, a correction to the activation energy due to the $\Pi$-variation amounts to $\delta F_{\text {barr }} \sim\left|\Pi-\Pi_{0}\right| V_{d}$; hence, the relative correction $\delta F_{\text {barr }} / F_{\text {barr }} \sim p a_{0} / \Lambda$ is very small indeed. The osmotic pressure variation can be, therefore, neglected.

In the general case, the energy barrier $F_{\text {barr }}$ depends on the difference between the local solvent pressure $P$ inside the core and the capillary pressure $P_{\text {cap }}=\gamma / a$,

$$
\begin{aligned}
& F_{\text {barr }} \simeq 2 \pi^{2} a_{0}^{4}\left(P_{\text {cap }}-P\right)^{2} / \gamma, \quad P_{\text {cap }}>P, \\
& F_{\text {barr }}=0, \quad P_{\text {cap }}<P .
\end{aligned}
$$

Equation (24) simply reflects the fact that the effective force opposing the droplet formation is the difference between the solvent pressures inside the droplet and in the core. The activation barrier vanishes if the solvent pressure in the core overcomes the opposing capillary pressure: in this case, the solvent freely leaks out of the core.

The droplet growth kinetics is studied in Sec. IV for the case of high $F_{\text {barr }}$.

\section{HIGH ACTIVATION BARRIER}

Let us first analyze the case of high activation energy $F_{\text {barr }} \gg T$ when a single isolated annular droplet formation is expected. As a result the jet core becomes slightly inhomogeneous with $a_{\text {core }}=a(z),\left|a-a_{0}\right| \ll a_{0}$. The rod contribution to the core free energy in this case can be written as

$$
\mathcal{F}_{\text {rod }}=\int F_{\text {rod }}(c) \pi a^{2}(z) \mathrm{d} z+\pi K \int\left(a_{z}^{\prime}\right)^{2} \mathrm{~d} z,
$$

where $F_{\text {rod }}(c)$ is defined in Eq. (2), $K$ is a phenomenological constant analogous to the Frank splay modulus for nonuniform nematic liquids [71], $c=c_{0}\left(a_{0}^{2} / a^{2}(z)\right)$ is the local concentration of rods defined by the rods number concentration in the absence of their axial motions [these motions can be neglected since the time of droplet formation, $\tau^{*}(\Lambda)$, cf. Eq. (42), is 
much shorter than $\left.\tau_{\mathrm{PR}}\right]$. The higher order terms in gradients of $a(z), a_{z}^{\prime}, a_{z z}^{\prime \prime}=\partial^{2} a / \partial z^{2}, \ldots$ are neglected here. The force balance at the jet surface now reads [cf. Eq. (14)]

$$
P-\frac{1}{2 \pi a(z)} \frac{\delta \mathcal{F}_{\text {rod }}}{\delta a(z)}=P_{\text {cap }},
$$

where $P_{\text {cap }} \simeq \gamma / a_{0}$ is capillary pressure and the variational derivative $\delta \mathcal{F}_{\text {rod }} / \delta a(z)$ is obtained using Eq. (25) and taking into account that $c=c(z)$ depends on the local radius $a(z)$,

$$
\frac{1}{2 \pi a(z)} \frac{\delta \mathcal{F}_{\text {rod }}}{\delta a(z)}=-\Pi-\frac{K a^{\prime \prime}}{a} .
$$

Here, $\Pi$ is defined in Eq. (4), and we set $\Pi \simeq \Pi_{0}$. This constant osmotic pressure approximation is justified below Eq. (23a); moreover, below Eq. (41), we also explicitly show that a correction to $\Pi_{0}$ is negligible. The above equations thus yield the following equation on the core profile $a(z)$ :

$$
P+\Pi_{0}+\frac{K a^{\prime \prime}}{a_{0}} \simeq \frac{\gamma}{a_{0}} .
$$

Far from the droplet, the core radius $a \rightarrow a_{0}$ and the pressure tends to

$$
P(|z| \rightarrow \infty) \equiv P_{0}=\gamma / a_{0}-\Pi_{0}
$$

Let us now turn to the growth kinetics of the droplet with $V_{d}>V_{d}^{*}$. The droplet is located at $z=0$, and its growth is provided by solvent current from both sides of the jet

$$
\mathrm{d} V_{d} / \mathrm{d} t=-2 J_{0}
$$

where $J_{0}(t)=\lim _{z \rightarrow+0} J(z, t)$ and $J=\pi a^{2} v$ [here $v=v(z, t)$ is the solvent velocity; it is also assumed that the rod volume fraction is small, $\phi \ll 1]$. Note that $J(z, t)<0$ near the droplet at $z>0$. Also note that here we focus on length scales $\gg a$, so $z \rightarrow+0$ is in fact equivalent to $z=\lambda / 2$, where $\lambda \simeq 2 \pi a$ is the droplet size along the jet axis.

The solvent current $J=\pi a^{2} v$ is generated by the solvent pressure gradient; with immobile rods, we get

$$
v=-\frac{1}{\zeta_{\|} c L} \frac{\partial P}{\partial z}, \quad J \simeq-\frac{\pi a_{0}^{2}}{\zeta_{\|} c_{0} L} \frac{\partial P}{\partial z} .
$$

In the last equation, it is taken into account that $a \simeq a_{0}$ and $c \simeq c_{0}$. Furthermore, the solvent pressure is defined in Eq. (28a), so

$$
P \simeq P_{0}-\frac{K}{a_{0}} \frac{\partial^{2} a}{\partial z^{2}}
$$

The rate of change of the local jet radius $\partial a / \partial t$ is defined by the continuity equation (coming from the jet incompressibility)

$$
\frac{\partial}{\partial t}\left(\pi a^{2}\right) \simeq-\frac{\partial J}{\partial z}
$$

Using Eqs. (30)-(32), we get the dynamical equation for the core radius $a=a(z, t)$,

$$
\frac{\partial a}{\partial t}=-\kappa \frac{\partial^{4} a}{\partial z^{4}}, \quad \kappa=\frac{K}{2 \zeta_{\|} c_{0} L}=\frac{K \xi^{2}}{2 \zeta_{\|}} .
$$

In the above equation, we used the relationship $L c_{0}=\xi^{-2}$. Equation (33) is supplemented by the boundary conditions

$$
\begin{gathered}
a \rightarrow a_{0} \text { at } z \rightarrow \infty \\
P \rightarrow P_{0}=\gamma / a_{0}-\Pi_{0} \text { at } z \rightarrow \infty \\
P=\frac{\gamma}{a_{0}}-\frac{\gamma V_{d}}{\left(2 \pi a_{0}^{2}\right)^{2}} \text { at } z=0 \\
\partial a / \partial z=0 \text { at } z=0
\end{gathered}
$$

Equation (34b) comes from Eq. (28b), Eq. (34c) reflects the force balance at the droplet surface $[P=\gamma C$ with $C$ defined in Eq. (19)], and Eq. (34d) comes from the force balance at the core/droplet interface, which reads $\delta \mathcal{F}_{\text {rod }} / \delta a(z)=0$ for $z=0$, and the condition $\Lambda \gg a_{0}$. Using Eqs. (29), (30), and (34c), we obtain

$$
\frac{\partial P}{\partial t}=-\frac{\gamma}{2 \pi a_{0}^{2} \zeta_{\|} c_{0} L} \frac{\partial P}{\partial z} \text { at } z=0
$$

The system of linear Eqs. (31), (33), (34a), (34b), (34d), and (35) can be solved analytically by using substitutions $P(z, t)=P_{0}+\Delta P(z, t), a(z, t)=a_{0}-\Delta a(z, t)$ and presenting the perturbation functions $\Delta P(z, t), \Delta a(z, t)$ for $z>0$ in the form (note that both functions are even in $z$ and vanish at $z \rightarrow \infty)$

$$
\begin{aligned}
& \Delta P(z, t)=e^{\alpha t}\left(\tilde{C} e^{-k z}+\tilde{C}^{*} e^{-k^{*} z}\right), \\
& \Delta a(z, t)=\frac{a_{0}}{K} e^{\alpha t}\left(\frac{\tilde{C}}{k^{2}} e^{-k z}+\frac{\tilde{C}^{*}}{k^{* 2}} e^{-k^{*} z}\right),
\end{aligned}
$$

where $\alpha, \tilde{C}$, and $k$ are constants and $\tilde{C}^{*}, k^{*}$ are complex conjugates of $\tilde{C}, k$. The constants must satisfy the following conditions: $\quad \alpha=-\kappa k^{4}, \quad \operatorname{Re}(k)>0, \quad \operatorname{Re}(\tilde{C} / k)=0, \quad$ and $\alpha \operatorname{Re}(\tilde{C})=\gamma \xi^{2} \operatorname{Re}(\tilde{C} k) /\left(2 \pi a_{0}^{2} \zeta_{\|}\right)$. After some algebra, we get

$$
\tilde{C}=\text { const. } \cdot e^{-i \pi / 4}, \quad k=\beta \sqrt{2} e^{i \pi / 4}
$$


Here, const. is a real constant and

$$
\beta=\frac{1}{\sqrt{2}}\left(\frac{\sqrt{2} \gamma}{\pi a_{0}^{2} K}\right)^{1 / 3}, \quad \alpha=4 \kappa \beta^{4}=\frac{\gamma \beta \xi^{2}}{\pi \zeta_{\|} a_{0}^{2}} .
$$

The solvent pressure then reads

$$
\begin{aligned}
P(z, t) & =P_{0}-\frac{\gamma}{a_{0}} A(t) \sqrt{2} e^{-\beta z} \cos \left(\beta z+\frac{\pi}{4}\right), \\
A(t) & =e^{\alpha\left(t-t_{0}\right)}
\end{aligned}
$$

where $t_{0}$ is an arbitrary time-shift, and the factor $\gamma / a_{0}$ is introduced for convenience [so the constant in Eq. (37) equals to const $\left.=\left(\gamma / a_{0}\right) e^{-\alpha t_{0}}\right]$. Time $t_{0}$ is connected with the magnitude of the original fluctuation [deviation of $V_{d}$ from $V_{d}^{*}$ at $t=0$, see Eq. (40)] whose evolution results in the droplet formation; $t_{0}$ is generally on the order of the time scale $\tau^{*}=1 / \alpha$. Using Eqs. (39) and (34c), we get the timedependence of the droplet volume $V_{d}$,

$$
V_{d}(t)=V_{d}^{*}+(2 \pi)^{2} a_{0}^{3} A(t),
$$

where $V_{d}^{*}$ is the critical volume of the droplet nucleus, Eq. (21), $V_{d}^{*} \ll a_{0}^{3}$. The radius of the jet core is given by

$$
a(z, t)=a_{0}-h(t) \sqrt{2} e^{-\beta z} \sin \left(\beta z+\frac{\pi}{4}\right)
$$

where the shrinkage depth $h(t)$ is $h(t) \simeq \pi \beta a_{0}^{2} A(t)$. We are now in a position to estimate the effect of the osmotic pressure variation. According to Eq. (23b), the variation is $\quad\left|\Pi-\Pi_{0}\right| \sim \Pi_{0} h / a_{0}, \quad$ hence $\quad\left|\Pi-\Pi_{0}\right| \sim \Pi_{0} A(t) a_{0} / \Lambda$. The characteristic amplitude of the solvent pressure variation is $\left|P-P_{0}\right| \sim A(t) \gamma / a_{0} \quad$ [cf. Eq. (39)], hence $\left|\Pi-\Pi_{0}\right| /\left|P-P_{0}\right| \sim p a_{0} / \Lambda \ll 1$, and, therefore, the osmotic pressure variation can be totally neglected.

The above equations are based on the linearized Eq. (19) for the droplet surface curvature. Therefore, they are valid in the regime of sufficiently small droplet with height $H \ll a_{0}$ and volume $V_{d} \ll a_{0}^{3}$. Using Eq. (40), we arrive at the condition $A(t) \ll 1$ which is roughly equivalent to $\alpha\left(t_{0}-t\right)>1$. Time $t_{0}$ is thus a crossover time demarcating the exponential growth regime (development of linear instability at $t<t_{0}$ ) and a later stage at $t>t_{0}$. The exponential growth stage is characterized by the time scale $\tau^{*}=1 / \alpha$ and length-scale $\Lambda=1 / \beta$. Using Eq. (38), we get

$$
\begin{aligned}
\Lambda & =\sqrt{2}\left(\frac{\pi a_{0}^{2} K}{\sqrt{2} \gamma}\right)^{1 / 3}, \\
\tau^{*} & =\tau^{*}(\Lambda)=\Lambda^{4} /(4 \kappa)=\frac{\pi \zeta_{\|} a_{0}^{2} \Lambda}{\gamma \xi^{2}} \sim \frac{\eta_{s} a_{0}^{2} \Lambda}{\gamma \xi^{2}} .
\end{aligned}
$$

With $\Lambda \leq L$, we arrive at $\tau^{*} \ll \tau_{P R} \sim\left(\eta_{s} a_{0} L^{2} / \gamma \xi^{2}\right)$.

The solvent pressure profile, Eq. (39) shows an absolute minimum $P_{\min }=P_{0}-\left(\gamma / a_{0}\right) A(t)$ at $z=0$ and a maximum $P_{\max } \approx P_{0}+0.2\left(\gamma / a_{0}\right) A(t)$ at $z=z_{c} \approx 1.6 \Lambda$ (Fig. 3). The

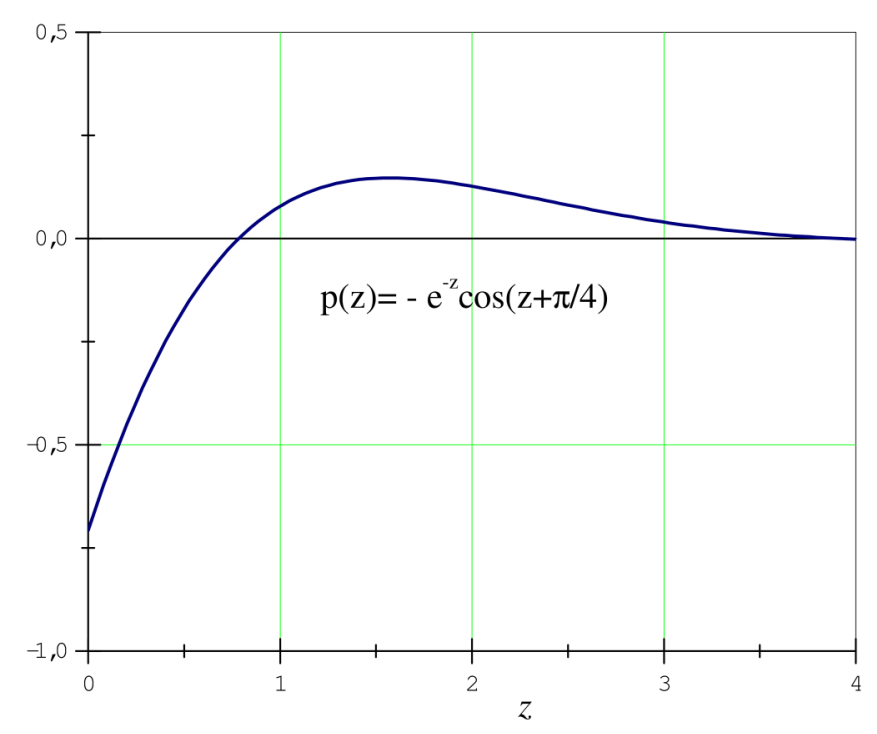

FIG. 3. Plot of the function $p(z)$, which defines the solvent pressure increment along the jet.

pressure $P_{\max }$ increases in time; it exceeds $P_{\text {cap }} \simeq \gamma / a_{0}$ for $t>t_{c}$, where the critical time $t_{c}$ is defined by

$$
\frac{\gamma}{a_{0}} A\left(t_{c}\right) \approx 5 \Pi_{0}
$$

Note that $A\left(t_{c}\right) \ll 1$ since $\Pi_{0} \ll \gamma / a_{0}$, so $t_{c}$ falls in the linear instability regime where the theory developed above is applicable. As has been discussed at the end of Sec. III [cf. Eq. (23b)], the condition $P\left(z_{c}\right)>P_{\text {cap }}$ for $t>t_{c}$ means that a new (daughter) solvent droplet must start to grow spontaneously at this point (i.e., at $z=z_{c}$ for $t>t_{c}$ ). The volume of the mother droplet (located at $z=0$ ) at the onset moment $t_{c}$ is $V_{d} \approx 6 V_{d}^{*}$ and its height is $H \approx 6 H^{*} \ll a_{0}$. Thus, each droplet generated by thermal activation triggers formation of 2 daughter (satellite) droplets on its side at a distance $\sim \Lambda$. This process takes a time $\sim \tau^{*}$, which is much shorter than the PR time. The new droplets, in turn, generate further droplets.

To summarize, the droplet nucleation is followed by an exponential growth of both the droplet height and volume: $H / H^{*}-1 \propto \exp \left(t / \tau^{*}\right)$, where $H^{*}$ is the critical droplet height. The upper bound estimate for the growth time is $\tau^{*} \sim \tau^{*}(L)$. The rate of droplet nucleation per jet segment of length $\sim \lambda$ is $f \sim E^{-1} / \tau^{*}$, where $E=\exp \left(F_{\mathrm{bar}} / T\right) \gg 1$. The rare droplets formed this way then induce nucleation of daughter droplets nearby. This mechanism provides self-replication of droplets at an early stage of their growth. The droplet concentration then increases from $c \sim(E \lambda)^{-1}$ (single droplets separated by distance $E \lambda$ ) to $c \sim(E \lambda)^{-1}(L / \lambda)$ (groups of droplets) during a time $t \sim \tau^{*}$.

\section{LOW ACTIVATION BARRIER}

In Sec. IV, we analyzed the process of a single annular droplet formation in the case of high activation barrier. The rate of single annular droplet nucleation per jet segment length $\sim \lambda=2 \pi a_{0}$ is $f \sim E^{-1} / \tau^{*}$, where $E=\exp \left(F_{\text {bar }} / T\right)$ and $\tau^{*}=\tau^{*}(L) \sim\left(\eta_{s} a_{0}^{2} L / \gamma \xi^{2}\right)$. The nucleation is followed by 
the droplet growth with the characteristic growth time $\sim \tau^{*}$ and is accompanied by a slight thinning of the jet in the region of length $\sim L$ around the nucleation site. It was implicitly assumed that no more than one droplet can be formed in this region during the characteristic time $\tau^{*}$. The latter condition is satisfied if

$$
f \tau^{*} L / \lambda \ll 1
$$

which is equivalent to $E \gg L / \lambda$, corresponding to

$$
F_{\text {bar }}>T \ln \left(L / a_{0}\right)
$$

In this case, the self-nucleated (parent) droplets are far from each other and do not interact, and the same is true for their respective families of inducted droplets.

For lower nucleation energy barrier, $E \ll L / \lambda$, the situation is different. Here, the typical concentration of nuclei (their number per unit length) is

$$
c_{n} \sim \frac{1}{\lambda} e^{-F_{\mathrm{bar}} / T}
$$

(the probability that a given $\lambda$-segment is occupied by a nucleus is $\sim 1 / E$ ), so the typical distance between the neighboring nuclei is $\Lambda \sim 1 / c_{n} \sim E \lambda \ll L$. This means that many $(L / \Lambda \gg 1)$ droplets nucleate almost simultaneously; hence, the relevant nucleation time is now

$$
\tau^{*}(\Lambda)=\frac{\pi \zeta_{\|} a^{2} \Lambda}{\gamma \xi^{2}} \sim \frac{\eta_{s} a_{0}^{2} \Lambda}{\gamma \xi^{2}} \ll \tau^{*}(L) \ll \tau_{\mathrm{PR}} .
$$

Note that this nucleation time does not involve any exponential (Arrhenius) factor: the energy penalty $F_{\text {bar }}$ is compensated by the ideal-gas entropic gain $T \ln (\Lambda / \lambda)$ per nucleating droplet.

Let us consider the postnucleation growth of solvent annuli located at positions $z_{n}$ in the linear regime assuming that sizes of the annular droplets $H_{n}(t)$ are different, $H_{n} \ll a$ [their volumes are $V_{n}(t) \simeq 2 \pi^{2} a_{0}^{2} H_{n}(t)$, where $n$ is an integer counting them]. The growth kinetics of the $n$th droplet volume is governed by the following equation:

$$
\frac{\mathrm{d} V_{n}}{\mathrm{~d} t}=J\left(z_{n}-0\right)-J\left(z_{n}+0\right)
$$

Here, $J(z) \approx \pi a^{2} v(z)$ is the solvent current through jet cross section at axial position $z$ and $J\left(z_{n}+0\right), J\left(z_{n}-0\right)$ are the local currents on both sides of the $n$th droplet; $v(z)$ is the solvent axial velocity (we assume that polymer volume fraction is small, $\phi \ll 1)$. The velocity in the core sections between the droplets depends on the local pressure gradient

$$
v(z)=-\frac{1}{\zeta_{\|} c L} \frac{\partial P}{\partial z}
$$

In our case, the radius of the jet core $a=a(t)$ is uniform ( $a$ does not depend on the coordinate $z$ since $\Lambda \ll L$ ); therefore, the continuity equation [Eq. (32)] in a section $z_{n}<z<z_{n+1}$ transforms to the following equation for the pressure $P(z)$ :

$$
\frac{\partial^{2} P}{\partial z^{2}}=\frac{2 \zeta_{\|}}{a_{0} \xi^{2}} \frac{d a}{d t}
$$

Note that $a \simeq a_{0}$ and $c \simeq c_{0}$ since $\left|a-a_{0}\right| \sim H \lambda /$ $\Lambda \ll H \ll a$. Equation (50) is supplemented by the boundary conditions, $P\left(z_{m}\right)=P_{m}$, where

$$
P_{m} \simeq \gamma\left(\frac{1}{a_{0}}-\frac{H_{m}}{2 a_{0}^{2}}\right)
$$

From (50) and (51), one gets the pressure profile in the section $z_{n}<z<z_{n+1}$ in terms of $H_{n}(t)$ and $\mathrm{d} a / \mathrm{d} t$,

$$
\begin{aligned}
P(z, t)= & \frac{\zeta_{\|}}{a_{0} \xi^{2}} \frac{\mathrm{d} a}{\mathrm{~d} t}\left(z-z_{n}\right)\left(z-z_{n+1}\right) \\
& +\gamma\left(\frac{1}{a_{0}}-\frac{H_{n}}{2 a_{0}^{2}}+\frac{\left(H_{n}-H_{n+1}\right)\left(z-z_{n}\right)}{2 a_{0}^{2}\left(z_{n+1}-z_{n}\right)}\right) .
\end{aligned}
$$

Now the rate of change of the droplets heights, $\mathrm{d} H_{n}(t) / \mathrm{d} t$, is coupled to the pressure and can be found using Eqs. (48) and (49). The remaining unknown function in Eq. (52) is the rate of change of the core radius, $\mathrm{d} a / \mathrm{d} t$. It can be found by using the energy-dissipation balance equation [68,72]

$$
\frac{\mathrm{d} \mathcal{F}}{\mathrm{d} t}+\dot{\mathcal{D}}=0
$$

Here, the change of the free energy $\mathcal{F}$ is defined by the total surface area reduction and the osmotic energy increment,

$$
\mathcal{F} \simeq-\frac{\pi^{2} \gamma}{2} \sum_{n}\left(H_{n}-H^{*}\right)^{2}=\sum_{n} \mathcal{F}_{n}
$$

where each term $\mathcal{F}_{n}$ in the sum is defined using Eq. (20) with $V_{d} \simeq 2 \pi^{2} a_{0}^{2} H_{n}$ and with constant shift $\left(\mathcal{F}_{n}=\Delta \mathcal{F}+\right.$ const. $)$; $H^{*}$ is defined in Eq. (21). The total dissipation rate $\dot{\mathcal{D}}$ arises due to friction between the solvent and immobile rods,

$$
\begin{aligned}
\dot{\mathcal{D}} & \simeq \pi a_{0}^{2} c_{0} L \zeta_{\|} \sum_{n} \int_{z_{n}}^{z_{n+1}} v_{n, n+1}^{2} \mathrm{~d} z \\
& \simeq \frac{\pi a_{0}^{2} \xi^{2}}{\zeta_{\|}} \sum_{n} \int_{z_{n}}^{z_{n+1}}\left(\frac{\partial P_{n, n+1}}{\partial z}\right)^{2} \mathrm{~d} z .
\end{aligned}
$$

Summation in (54a) and (54b) runs over all the droplets.

A further analysis of the above equations [Eqs. (48)-(54a) and (54b)] is simplified by assuming that the annular droplets are equidistant (an infinite sequence of droplets arranged with spacing $\Lambda, z_{n}=n \Lambda$ ). In this case, the linearized master 
equation for the droplet heights reads

$$
\frac{\mathrm{d} H_{n}}{\mathrm{~d} t}=\frac{1}{\tau^{*}(\Lambda)}\left[\left(2 H_{n}-H_{n-1}-H_{n+1}\right) / 4+3\left(\bar{H}-H^{*}\right)\right],
$$

where $\bar{H}=\bar{H}(t)=\sum_{n} H_{n} / \sum_{n} 1$ is the average height of the droplets, and the characteristic time $\tau^{*}(\Lambda)$ is defined in Eq. (47). The general solution of this equation is

$$
\begin{aligned}
H_{n}= & H^{*}+h_{0} \exp \left(v_{0} t\right) \\
& +\int_{0}^{1 / 2} h(k) \cos (2 \pi k n) \exp (v(k) t) \mathrm{d} k
\end{aligned}
$$

where $h_{0}$ and $h(k)$ are the initial amplitudes of the uniform and nonuniform linear modes and

$$
v_{0}=3 / \tau^{*}(\Lambda), \quad v(k)=\frac{1-\cos (2 \pi k)}{2 \tau^{*}(\Lambda)}
$$

are their growth rates [here, $k$ is the wave number and $v(k)$ is the dispersion function]. The fastest growing nonuniform mode corresponds to $k=1 / 2$ with the growth rate $v(1 / 2)=1 / \tau^{*}(\Lambda)$. This mode reflects the straightforward solvent exchange between the neighboring droplets, leading to alternatively bigger (say, even) and smaller (say, odd) droplets: $H_{n}-\bar{H} \propto(-1)^{n} \exp \left(t / \tau^{*}(\Lambda)\right)$. By contrast, the uniform mode grows due to solvent release from the core. Its growth rate is high: $v_{0} \geq 3 v(k)$. As a result, the droplet size distribution $\left\{H_{n}\right\}$ tends to rapidly converge to the stable uniform mode

$$
H_{n} \rightarrow \bar{H}=H^{*}+h_{0} \exp \left(v_{0} t\right)
$$

with periodic pressure profile: $P(z+\Lambda)=P(z)$,

$$
P(z, t) \simeq \gamma\left(\frac{1}{a_{0}}-\frac{\bar{H}(t)}{2 a_{0}^{2}}\right)+\frac{3 \gamma\left(\bar{H}(t)-H^{*}\right)}{\Lambda^{2} a_{0}^{2}} z(\Lambda-z), \quad 0<z<\Lambda
$$

The pressure shows maximums $P=P_{\max } \simeq \gamma\left(\left(1 / a_{0}\right)-\right.$ $\left.\left(\bar{H}(t) / 2 a_{0}^{2}\right)\right)+\left(3 \gamma\left(\bar{H}(t)-H^{*}\right) / 4 a_{0}^{2}\right)$ at midpoints between the droplets, $z=(n+(1 / 2)) \Lambda$. As soon as $P_{\max }$ exceeds $P_{\text {cap }}=(\gamma / a) \simeq\left(\gamma / a_{0}\right)$, the solvent must leak out of the core forming a new droplet. This happens for $H>3 H^{*}$. As a result, the pressure $P$ there decreases below $P_{\text {cap }}$ and the number of droplets doubles. This mechanism of droplet induction (which is similar to that considered in Sec IV) leads to a reduction of the mean distance $\Lambda$ between the droplets by a factor of 2 during time $t_{d} \sim \tau^{*}(\Lambda)$. The rate of change of $\ln (\Lambda)$ can be estimated as

$$
-\frac{\mathrm{d} \ln (\Lambda)}{\mathrm{d} t} \simeq \frac{\ln (2)}{t_{d}} \sim \frac{1}{\tau^{*}(\Lambda)} \sim \frac{w}{\Lambda}
$$

where $w=L / \tau^{*}(L) \sim\left(\gamma \xi^{2} / \eta_{s} a_{0}^{2}\right)$. It leads to

$$
\Lambda \simeq \Lambda_{0}-w t
$$

where $\Lambda_{0} \sim E \lambda$. Hence, $\Lambda$ decreases to the minimal value $\left(\Lambda_{\text {min }} \simeq \lambda=2 \pi a\right)$ during the replication time

$$
\tau_{r} \sim(E-1) \lambda / w \sim \tau^{*}\left(\Lambda_{0}\right)
$$

A further droplet growth proceeds with constant number of droplets (separated by the distance $D \sim \Lambda_{\min } \simeq \lambda$ ). The droplets then grow approximately exponentially according to Eq. (55) with the characteristic time $\tau^{*}(\Lambda)$ replaced with $\tau^{*}\left(\Lambda_{\min }\right) \equiv \tau_{\min }^{*}$, where

$$
\tau_{\min }^{*} \sim \tau^{*}\left(a_{0}\right) \sim \frac{\eta_{s} a_{0}^{3}}{\gamma} \frac{\phi_{0}}{d^{2}}
$$

In the case of even low barrier, $F_{\mathrm{bar}} / T \leq 1$, the droplets nucleate already at a maximum concentration (with the minimal separation $\Lambda \sim \Lambda_{\min }$ ), so the replication stage (introduction of new droplets between the existing growing droplets) is forbidden. Assuming equidistant droplets as before, we then arrive at the exponential droplet growth kinetics [cf. Eq. (57)] $H_{n}-H^{*} \propto \exp \left(3 t / \tau_{\min }^{*}\right)$. Note that Eq. (57) is not applicable during the replication stage in the activated regime, $F_{\text {bar }} \gg T$, where the droplet growth is faster than exponential due to a decrease of $\Lambda$ and $\tau^{*}(\Lambda)$ in time.

\section{NONLINEAR GROWTH REGIME}

The linear regime of exponential growth ends up when the mean droplet height $H$ becomes comparable with $a_{0}$. At this point, roughly half of the solvent is already squeezed in the droplets (as the droplet separation is $\sim \lambda=2 \pi a_{0}$ ). In reality, the annular droplets are not exactly equidistant and their sizes are different: the system of droplets is polydisperse. The core thinning (driven by the capillary forces) continues in the nonlinear regime with essentially the same time scale as for the linear regime $\left(\sim \tau_{\text {min }}^{*}\right)$. Before turning to the kinetics of this process, let us describe the final stage of the system $(t \rightarrow \infty)$. It involves a compressed core of radius $a_{\infty}$ and a big solvent droplet (with $H \rightarrow \infty$ ) around the core. The terminal core size $a=a_{\infty}$ is defined by minimization of the total core free energy $F=F_{s}+F_{\text {rod }}$, where $F_{s}=\gamma A$, $A=2 \pi a L_{\text {tot }}$ is the core surface, $L_{\text {tot }}$ is the total core length (the droplet surface energy can be neglected; in addition, the length fraction of the core inside the droplet tends to 0 in this regime), and $F_{\text {rod }}$ is the total osmotic energy [cf. Eq. (2)]. Recalling that

$$
\frac{\partial F_{s}}{\partial V_{\text {core }}}=P_{\text {cap }}=\frac{\gamma}{a}, \quad \frac{\partial F_{\text {rod }}}{\partial V_{\text {core }}}=-\Pi,
$$

where $V_{\text {core }}=\pi a^{2} L_{\text {tot }}$ is the core volume and $\Pi$ is the osmotic pressure, we find that the equilibrium condition 
$(\min F)$ leads to the pressure balance $\Pi=P_{\text {cap }}$,

$$
\Pi a=\gamma
$$

The osmotic pressure $\Pi$ depends on the rod concentration $\phi=\phi_{0} a_{0}^{2} / a^{2} ; \Pi$ increases with $\phi$ at least as fast as $\phi$ (indeed, $\Pi=c T+\Pi_{\text {int }}$, where $\Pi_{\text {int }}$ is the osmotic pressure contribution due to steric repulsion, $\Pi_{\mathrm{int}} / c$ increases with $c$, cf. Sec. II). Therefore, $\Pi a$ increases as $\phi$ is increased (and $a$ is decreased). At $\phi \sim 0.5$, the osmotic pressure $\Pi \sim 4 c T \sim$ $T / d^{2} L \quad$ [cf. Eq. (6)], so $\Pi a / \gamma \sim\left(T / \gamma d^{2}\right) a / L$. With $a \ll a_{0} \ll L, d \sim 2 \mathrm{~nm}, \gamma \sim 100 \mathrm{dyn} / \mathrm{cm}$, and other parameters estimated below Eq. (23a), we get $\Pi a / \gamma \ll 10^{-2}$. Thus, $\Pi a \ll \gamma$ for $\phi \sim 0.5$, so condition (64) cannot be satisfied for any $\phi \leq 0.5$ : the condition demands that the core must be dense, $\phi \approx 1$. The terminal core radius, therefore, is

$$
a_{\infty} \approx a_{0} \sqrt{\phi_{0}}
$$

Note that $a_{\infty} \ll a_{0}$ since $\phi_{0} \ll 1$. It is, therefore, fair to conclude that a dense solidlike fiber with highly aligned rods must be formed at the late stage of capillary thinning. Most of the solvent is then released as surface droplets around the fiber. The fiber can still contain a certain amount of solvent (a fraction $1-\phi \sim 10 \%$ ) in the interstices between the rods.

Let us turn to the core-thinning process in the regime $a_{\infty} \leq a \ll a_{0}$. This process is driven by the capillary pressure; the osmotic pressure can be neglected since $\Pi \ll \gamma / a$ for $\phi \leq 0.5$. In the specified regime, the amount of the released solvent does not change much (it is always close to $100 \%$ ) and so does the droplet size $H$ (cf. the last part of this section). The droplet size $H$ is much larger than the core thickness, $H \gg a$, in this regime, hence the droplets are nearly spherical with radius $R \simeq H \sim a_{0}$ [we assume here that the number of droplets is conserved during the core-thinning stage as verified below Eq. (81b)]. As a result, the droplet pressure $\gamma / R$ can be neglected in comparison with the core capillary pressure $P_{\text {cap }}=\gamma / a$. The droplets are connected by core segments of length $\Lambda$ (see Fig. 4); $\Lambda$ can be obtained from the volume conservation, $\pi a_{0}^{2}(\Lambda+2 R)=(4 \pi / 3) R^{3}+\pi a^{2} \Lambda$, leading to

$$
\Lambda \simeq \frac{4 R^{3}}{3 a_{0}^{2}}
$$

Hence, $\Lambda \sim a_{0}$ as $R \sim a_{0}$ (here we consider droplets of similar size $R$ ).

A high pressure $P_{\text {cap }}$ inside a $\Lambda$-string generates the solvent flow toward its ends, leading to the core thinning with rate $\dot{\varepsilon}=-2(\partial / \partial t) \ln a$. The solvent velocity $v=v(x, t)$

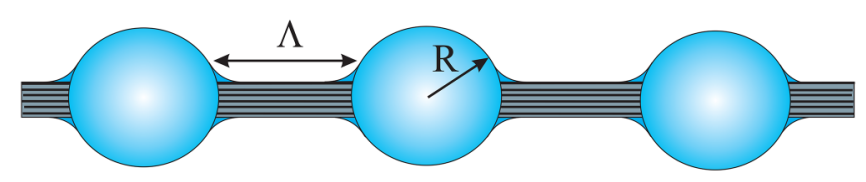

FIG. 4. Sequence of annular droplets of the radius $R \sim a_{0}$ on the jet core in the nonlinear regime when the core shrinks due to the Laplace pressure. along the string, $0<x<\Lambda$, is defined by the volume conservation equation

$$
\frac{\partial J}{\partial x}+\frac{\partial}{\partial t}\left(\pi a^{2}\right)=0
$$

where

$$
J=J(x, t)=\phi_{s}(t) \pi a^{2}(t) v(x, t)
$$

is the solvent current along the core axis and $\phi_{s}=1-\phi$ is the solvent volume fraction. Therefrom, we get

$$
J=\pi a^{2} \dot{\varepsilon}(x-\Lambda / 2), \quad v=\frac{\dot{\varepsilon}}{\phi_{s}}(x-\Lambda / 2)
$$

The rate $\dot{\varepsilon}$ can be obtained using the energy-dissipation balance Eq. (53), where the dissipation rate $\dot{\mathcal{D}}$ reads

$$
\dot{\mathcal{D}}=\int k_{f} v^{2} \mathrm{~d} V
$$

and $k_{f}=c L \zeta_{\|}=(4 / \pi) \zeta_{\|} \phi d^{-2}$ is the relevant friction constant. Using Eqs. (69) and (70a), we obtain

$$
\dot{\mathcal{D}} \simeq \frac{\pi}{12} k_{f} a^{2} \Lambda^{3}\left(\frac{\dot{\varepsilon}}{1-\phi}\right)^{2}
$$

where $k_{f} a^{2}=(4 / \pi) \zeta_{\|} d^{-2} \phi_{0} a_{0}^{2}$ does not change in time.

As for the free energy $\mathcal{F}$, it is dominated by the string surface energy, $\mathcal{F} \simeq 2 \pi a \Lambda \gamma$, so

$$
\frac{\mathrm{d} \mathcal{F}}{\mathrm{d} t}=-\pi \dot{\varepsilon} \gamma a \Lambda
$$

Note that the solvent is assumed to be good for the rods, so the rods are wetted by the solvent, and $\gamma$ is always close to the solvent/air surface tension, which is constant (independent of $\phi)$. Equations (53), (70b), and (71) lead to

$$
\dot{\varepsilon}=3 \pi \frac{\gamma d^{2}(1-\phi)^{2}}{\Lambda^{2} a \zeta_{\|} \phi}
$$

So far, we assumed monodisperse strings. In the case of a random string length, the factor $\Lambda^{2}$ in the above equation must be replaced with $\bar{\Lambda}^{2} \equiv\left\langle\Lambda^{3}\right\rangle /\langle\Lambda\rangle$, where the brackets $\langle\cdots\rangle$ signify the number average. Exactly the same result as Eq. (72) can be obtained by imposing the force balance on the core surface: the mean solvent pressure $P$ (averaged over all the strings) must be equal to $P_{\text {cap }}=\gamma / a$.

Taking into account that $\phi=\phi_{0} a_{0}^{2} / a^{2}$ leads to $\dot{\varepsilon}=$ $\mathrm{d}(\ln \phi) / \mathrm{d} t$ and $a=a_{\infty} / \sqrt{\phi}$, so Eq. (72) transform to

$$
\tau_{\mathrm{th}} \mathrm{d} \phi / \mathrm{d} t=(1-\phi)^{2} \sqrt{\phi}
$$


where

$$
\tau_{\text {th }}=\frac{\zeta_{\|} a_{0} \Lambda^{2} \sqrt{\phi_{0}}}{3 \pi \gamma d^{2}} \sim \frac{\eta_{s} a_{0}^{3} \sqrt{\phi_{0}}}{\gamma d^{2}}
$$

is the characteristic time. The solution of Eq. (73a) is

$$
\frac{\sqrt{\phi}}{1-\phi}+\frac{1}{2} \ln \frac{1+\sqrt{\phi}}{1-\sqrt{\phi}}=\frac{t}{\tau_{\text {th }}}+\text { const. }
$$

The result can be simplified in two asymptotic regimes, $t \ll \tau_{\text {th }}$ and $t \gg \tau_{\text {th }}$

$$
\begin{gathered}
\phi \simeq \begin{cases}\left(\frac{t}{2 \tau_{\mathrm{th}}}+\sqrt{\phi_{0}}\right)^{2}, & t \ll \tau_{\mathrm{th}}, \\
1-\frac{\tau_{\mathrm{th}}}{t}, & t \gg \tau_{\mathrm{th}},\end{cases} \\
a \simeq \begin{cases}a_{0}\left(1+\frac{t}{2 \tau_{\mathrm{th}} \sqrt{\phi_{0}}}\right)^{-1}, & t \ll \tau_{\mathrm{th}}, \\
a_{\infty}\left(1+\frac{\tau_{\mathrm{th}}}{2 t}\right), & t \gg \tau_{\mathrm{th}} .\end{cases}
\end{gathered}
$$

The core thinning, therefore, essentially follows the power law

$$
a-a_{\infty} \propto 1 / t
$$

At long times, when the osmotic pressure $\Pi$ becomes significant $(\Pi \sim \gamma / a$ ), this power law is followed by an exponential decay of $a-a_{\infty}$. The characteristic thinning time $\tau_{\text {th }}$ is much longer than the linear growth time $\tau_{\text {min }}^{*}: \tau_{\text {th }} \gg \tau_{\text {min }}^{*} \sim \tau_{\text {th }} \sqrt{\phi_{0}}$.

We are now in a position to consider the last stage of droplet growth well beyond $a_{0}$, where $H \sim R \gg a_{0}$. The corresponding time $\tau_{d}$ is much longer than $\tau_{\text {th }}$ [see below, Eq. (81b)], so we can simplify the analysis by assuming $\phi \approx 1, a \approx a_{\infty} \approx a_{0} \sqrt{\phi_{0}}$. As was mentioned above, the solvent droplets are actually polydisperse, but their total volume is nearly constant (since the core volume is negligible for $\left.a \ll a_{0}\right)$. The droplets grow in size and their number decreases according the coalescence mechanism similar to that proposed by Lifshitz and Slyozov [73]: the larger droplets grow while the smaller droplets shrink and disappear. Let $R$ be the typical droplet size. The length of the core strings between the droplets can be obtained using Eq. (66),

$$
\Lambda \sim R^{3} / a_{0}^{2} \gg R
$$

Therefore, only a small fraction of the core surface stays in contact with the solvent droplets. Note that while a droplet is nearly spherical (since $R \gg a$ ), its shape is distorted near the core: the droplets meet the core surface at a small contact angle.

The droplet volume changes due to solvent flow along the core strings. To describe it we keep using the continuous description, Eq. (68), assuming a small finite residual solvent fraction $\phi_{s}=1-\phi_{\infty}$. Let us consider one string of length $\Lambda$ between the drops $R_{1}$ and $R_{2}, R_{1}>R_{2}$ (Fig. 5). The solvent velocity $v$ is generated by the pressure gradient: $k_{f} v=-\partial P / \partial z$. The friction constant is $k_{f}=\zeta_{\|} c L \sim \zeta_{\|} / d^{2}$ since $\phi$ is close to 1 (note that the factor $\zeta_{\|}=2 \pi \eta_{s} / k_{H}$ may be enhanced due to a renormalization of the effective solvent viscosity $\eta_{s}$ in the dense state). The solvent pressure at the string ends $(z=0$ and $z=\Lambda)$ is defined by the capillary pressure: $P(0)=\gamma / R_{1}, P(\Lambda)=\gamma / R_{2}$. Therefore, $v=-\left(\Delta P / k_{f} \Lambda\right)$, where $\Delta P=\gamma\left(1 / R_{2}-1 / R_{1}\right)$. The solvent current $J \simeq \pi a_{\infty}^{2} \phi_{s} v$ is directed toward the first droplet. Its volume $V_{1}=(4 \pi / 3) R_{1}^{3}$ increases with the rate

$$
\mathrm{d} V_{1} / \mathrm{d} t=-J \simeq 4 \pi k_{J} \Delta P / \Lambda
$$

where $k_{J}=\phi_{s} a_{\infty}^{2} /\left(4 k_{f}\right)$. Setting $R_{1}=R, R_{2}=R / 2$ and recalling Eq. (79), we get

$$
R^{2} \mathrm{~d} R / \mathrm{d} t=k_{J} \Delta P / \Lambda \sim k_{J} \gamma a_{0}^{2} / R^{4}
$$

The solution is

$$
R \sim a_{0}\left(t / \tau_{d}\right)^{1 / 7}, \quad t \gg \tau_{d}
$$

with the characteristic droplet growth time

$$
\tau_{d} \sim \frac{a_{0}^{3} \zeta_{\|}}{\phi_{0} \phi_{s} \gamma d^{2}}
$$

Obviously $\tau_{d} \gg \tau_{\text {th }}$ since $\tau_{d} / \tau_{\text {th }} \sim \phi_{0}^{3 / 2} \phi_{s} \ll 1$. Therefore, the droplet size does not change significantly during the corethinning time $\tau_{\text {th }}$.

The assumptions made (the neglected effect of the second neighbor and assumed constant ratio $R_{1} / R_{2}=2$ ) do not affect the final scaling dependence for the droplet size (apart from a numerical prefactor which is omitted anyway). Thus, the droplet size increases very slowly according to the anomalous power law, $R \propto t^{1 / 7}$ for $t \gg \tau_{d}$. The kinetics of solvent release and fiber formation in the case of low activation energy, $F_{\text {bar }} \leq T$, thus involve three main stages: (i) exponential droplets growth (the height $H$ increases up to $H \sim a_{0}$ ) with time $\tau_{\min }^{*}$; (ii) core thinning and fiber formation (the core size decreases from $a_{0}$ to $a_{\infty}$ ) occurring with longer time $\tau_{\text {th }}$; and (iii) droplets coalescence and further growth (beyond $H \sim a_{0}$ ) with yet longer characteristic time $\tau_{d}$.

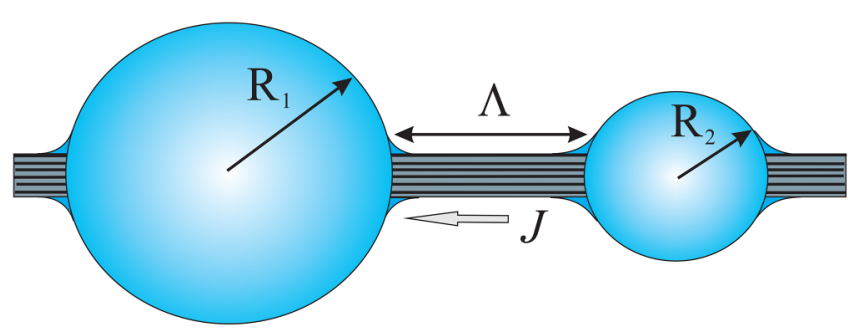

FIG. 5. Coalescence of droplets in the terminal stage of solvent/rods separation. 


\section{CONCLUDING REMARKS}

In the present paper, we examined multiple annular droplets formation in the liquid jet formed by dispersion of rigid rods. We focused on the most interesting regime when the jet thickness is smaller than the rod length and the rods are highly oriented. This regime appears in the terminal stage of the pinching-off dynamics of the liquid bridge.

We show that the rods get effectively trapped inside the jet core, whereas the solvent drains to the surface and forms annular droplets there. Depending on the polymer concentration, the formation of solvent annuli can occur either by means of an activation mechanism or like a spinodal decomposition. First, the annular droplets grow exponentially; then, the growth slows down and the jet core shrinks up to the minimum value at which the capillary forces are in balance with the osmotic pressure. Afterward, the droplets grow slowly by coalescence. The explored process of the capillarydriven phase separation occurs much faster than the PR pinching instability, so the separation effect can prevent the jet from breaking up. This mechanism differs from the mechanism of phase separation, which is connected with a reduction of the steric repulsion of the stretched chains [47-49]. Thereby, the discovered capillary-driven phase separation process provides a rather universal mechanism of fiber formation in dispersions of rodlike particles.

The theory developed here is hinged on a number of conditions. The main conditions required for the theory to be rigorous are listed as follows:

1. We consider solutions of absolutely rigid and long macromolecules (stiff rods) of length $L \gg d$ (more precisely, we also assume that $l_{p} \gg L$, where $l_{p}$ is the macromolecular persistence length).

2. The concentration $c$ of rods is such that their volume fraction $\phi$ is small, $\phi \ll 1$, but $c L^{3} \gg 1$. The first condition provides simpler and richer nonlinear dynamics with wellseparated time scales (cf. Sec. VI); it is also used in Sec. IV. The second condition leads to a high solution viscosity, $\eta \gg \eta_{s}$, required to neglect inertial effects (see points 3 and 5 below). The two conditions still allow for a wide concentration range, $(d / L)^{2} \ll \phi \ll 1$.

3. The jet flow of the solution, which is thinning due to capillary forces, must fall in the viscous regime. This requirement should actually apply only to the relevant (the thinnest) part of the jet of length $L_{z}$ we consider. It demands that the jet radius $a$ is small enough, $a \ll a^{*}$, where $a^{*}=\eta^{2} / \rho \gamma$, and that $L_{z} \ll \sqrt{a a^{*}}$. These conditions are not so restrictive in the case of long rods (cf. Sec. II D) as the elongational viscosity $\eta$ of the solution is strongly enhanced due to the rodlike solute: $\eta$ is proportional to $c L^{3}$, hence $a^{*}$ scales as $L^{6}$.

4. We consider a thin jet whose radius $a$ is much smaller than the rod length $L: a \ll L$. This condition implies that the solute molecules are long; it is pretty compatible with the conditions $L \gg d$ and $a \ll a^{*}$ mentioned above. The regime $a \ll L$ emerges naturally in the course of continuous viscocapillary thinning occurring with the rate $d a / d t \sim-\gamma / \eta$. The condition $a \ll L$ is very important: it ensures that the characteristic time scale of droplet formation $\tau^{*}$ is much shorter than the PR time $\tau_{\mathrm{PR}}, \tau^{*} \ll \tau_{\mathrm{PR}}$ [cf. Sec. IV below Eq. (42) and Eq. (47)]. Therefore, the new mechanism of capillary-driven droplet formation elucidated in this paper can be faster than (can preempt) the classical PR thinning and breakup.

5. To be able to neglect the inertial flow effects in the thinning jet we have to demand $a^{*} a \gg L^{2}$. Together with condition $a \ll a^{*}$, it implies that $a^{*} \gg L$, which is quite realistic with $\eta>1 \mathrm{P}$ [such viscosity can be achieved, for example, with $c L^{3}>100$, cf. Eq. (13)] and $L$ in the micrometer scale.

6. The last condition, which is central to the theory, says that the osmotic pressure of rods, $\Pi$, is much lower than capillary pressure $\gamma / a: p \equiv \Pi a / \gamma \ll 1$. It ensures that the critical toroidal droplet (in the activated state) is much thinner than the core and justifies the perturbation approach adopted in Sec. III. This condition is also quite realistic as estimated in Sec. III above Eq. (14). Interestingly, the very same condition ensures that rods are highly oriented by the capillary-driven elongational flow [cf. Eq. (12) and note that in the regime of interest $\eta / \eta_{s} \sim c L^{3}$ and $\left.\Pi \sim c T\right]$.

The most important condition is $l_{p} \gg L \gg a$. The jet radius $a$ must exceed $\sim 10 \mathrm{~nm}$ to have many polymers in the jet cross section. Then, the above conditions demand that the rod length $L$ must be at least $\sim 100 \mathrm{~nm}$, and its persistence length $l_{p}$ must be larger than $\sim 300 \mathrm{~nm}$. The required rigidity is thus very high. It can be achieved for helical polymers (like $\mathrm{P} \gamma \mathrm{BG}$ ) and their bundles or for biological fibrils (protein polymers) mentioned below. Experimental observation and identification of the predicted solvent/rods demixing mechanism is, therefore, a challenging problem.

A thin quasiuniform jet can be easily produced, for example, by stretching of a liquid droplet. This process gives rise to formation of a thin neck (pinching region) whose length $L_{z}=L_{z}(t)$ and radius $a=a(t)$ decrease in time. The theory developed above is applicable to such pinching bridge with $a \ll L_{z}$, which can be locally considered as a uniform cylinder. One possibility to verify our results is to study dispersions of rigid nanorods. Such macromolecular rods (with length $\geq 1-10 \mu \mathrm{m}$ ) can be represented, for example, by protein polymers (F-actin, microtubules) and other selfassembling supramolecular structures (like triarylamine fibers [74-78]). The appropriate liquid bridges can also be formed by suspensions of carbon nanotubes [63-65]. Another opportunity is to use a polymer solution of very long and stiff macromolecules aligned by the flow. The proposed mechanism of capillary-induced phase separation can be relevant for numerous applications including fiber spinning for production of lightweight high-strength materials [56-58].

\section{ACKNOWLEGMENTS}

The work of A.V.S. was supported by the Russian Foundation for Basic Research (Grant No. 18-29-17002) and partially by CNRS (Contract No. 783221). A.N.S. acknowledges a partial support from the International Research Training Group (IRTG) "Soft Matter Science: Concepts for the Design of Functional Materials." 


\section{REFERENCES}

[1] Eggers, J., "Nonlinear dynamics and breakup of free-surface flows," Rev. Mod. Phys. 69, 865-929 (1997).

[2] Basaran, O. A., "Small-scale free surface flows with breakup: Drop formation and emerging applications," AIChE J. 48, 1842-1848 (2002).

[3] Villermaux, E., "Fragmentation," Ann. Rev. Fluid Mech. 39, 419-446 (2007).

[4] Eggers, J., and E. Villermaux, "Physics of liquid jets," Rep. Prog. Phys. 71, 036601 (2008).

[5] Kooij, S., R. Sijs, M. M. Denn, E. Villermaux, and D. Bonn, "What determines the drop size in sprays?," Phys. Rev. X 8, 031019 (2018).

[6] Malkin, A. Y., A. Arinstein, and V. G. Kulichikhin, "Polymer extension flows and instabilities," Prog. Polym. Sci. 39, 959-978 (2014).

[7] Kulichikhin, V. G., I. Y. Skvortsov, A. V. Subbotin, S. V. Kotomin, and A. Y. Malkin, "A novel technique for fiber formation: Mechanotropic spinning_Principle and realization," Polymers 10, 856-856 (2018).

[8] Xue, J., T. Wu, Y. Dai, and Y. Xia, "Electrospinning and electrospun nanofibers: Methods, materials, and applications," Chem. Rev. 119, 5298-5415 (2019).

[9] Rayleigh, L., "Instability of jets,” P. Lond. Math. Soc. 1, 4-13 (1878).

[10] Weber, C., "Zum zerfall eines flussigkeitsstrahle," Z. Angew. Math. Mech. 11, 136-154 (1931).

[11] Schulkes, R. M. S. M., "The contraction of liquid filaments," J. Fluid Mech. 309, 277-300 (1996).

[12] Chen, A. U., P. K. Notz, and O. A. Basaran, "Computational and experimental analysis of pinch-off and scaling," Phys. Rev. Lett. 88, 174501 (2002).

[13] Notz, P. K., and O. A. Basaran, "Dynamics and breakup of a contracting liquid filament," J. Fluid Mech. 512, 223-256 (2004).

[14] Driessen, T., R. Jeurissen, H. Wijshoff, F. Toschi, and D. Lohse, "Stability of viscous long liquid filaments," Phys. Fluids 25, 062109 (2013).

[15] Li, Y., and J. E. Sprittles, "Capillary breakup of a liquid bridge: Identifying regimes and transitions," J. Fluid Mech. 797, 29-59 (2016).

[16] Keller, J. B., and M. J. Miksis, "Surface tension driven flows," SIAM J. Appl. Math. 43, 268-277 (1983).

[17] Papageorgiou, D. T., "On the breakup of viscous liquid threads," Phys. Fluids 7, 1529-1544 (1995).

[18] Papageorgiou, D. T., "Analytical description of the breakup of liquid jets," J. Fluid Mech. 301, 109-132 (1995).

[19] Lister, J. R., and H. A. Stone, "Capillary breakup of a viscous thread surrounded by another viscous fluid,” Phys. Fluids 10, 2758-2764 (1998).

[20] Castrejón-Pita, J. R., A. A. Castrejón-Pita, S. S. Thete, K. Sambath, I. M. Hutchings, J. Hinch, J. R. Lister, and O. A. Basaran, "Plethora of transitions during breakup of liquid filaments," Proc. Natl. Acad. Sci. U.S.A. 112, 4582-4587 (2015).

[21] Kamat, P. M., B. W. Wagoner, S. S. Thete, and O. A. Basaran, "Role of Marangoni stress during breakup of surfactant-covered liquid threads: Reduced rates of thinning and microthread cascades," Phys. Rev. Fluids 3, 043602 (2018).

[22] Doshi, P., R. Suryo, O. E. Yildirim, G. H. McKinley, and O. A. Basaran, "Scaling in pinch-off of generalized Newtonian fluids," J. Nonnewton. Fluid Mech. 113, 1-27 (2003).

[23] Suryo, R., and O. A. Basaran, "Local dynamics during pinch-off of liquid threads of power law fluids: Scaling analysis and self-similarity," J. Nonnewton. Fluid Mech. 138, 134-160 (2006).

[24] Huisman, F. M., S. R. Friedman, and P. Taborek, "Pinch-off dynamics in foams, emulsions and suspensions," Soft Matter 8, 6767-6774 (2012).
[25] Denn, M. M., and J. F. Morris, "Rheology of Non-Brownian suspensions," Ann. Rev. Chem. Biomol. Eng. 5, 203-228 (2014).

[26] Daristotle, J. L., A. M. Behrens, A. D. Sandler, and P. Kofinas, "A review of the fundamental principles and applications of solution blow spinning,” ACS Appl. Mater. Interfaces 8, 34951-34963 (2016).

[27] Reneker, D. H., A. L. Yarin, E. Zussman, and H. Xu, "Electrospinning of nanofibers from polymer solutions and melts," Adv. Appl. Mech. 41, 43-195 (2007).

[28] McKinley, G. H., Visco-elasto-capillary thinning and break-up of complex fluids, in Rheological Review (The British Society of Rheology, Aberystwyth, 2005), pp. 1-48.

[29] Bazilevskii, A. V., S. I. Voronkov, V. M. Entov, and A. N. Rozhkov, "Orientation effects in the breakup of jets and threads of dilute polymer solutions," Sov. Phys. Dokl. 26, 333-336 (1981).

[30] Yarin, A. L., Free Liquid Jets and Films: Hydrodynamics and Rheology (Wiley, New York, 1993).

[31] Oliveira, M. S. N., and G. H. McKinley, "Iterated stretching and multiple beads-on-a-string phenomena in dilute solutions of highly extensible flexible polymers," Phys. Fluids 17, 071704 (2005).

[32] Clasen, C., J. Eggers, M. A. Fontelos, J. Li, and G. H. McKinley, "The beads-on-string structure of viscoelastic threads," J. Fluid Mech. 556, 283-308 (2006).

[33] Sur, S., and J. Rothstein, "Drop breakup dynamics of dilute polymer solutions: Effect of molecular weight, concentration, and viscosity," J. Rheol. 62, 1245-1259 (2018).

[34] Sattler, R., C. Wagner, and J. Eggers, "Blistering pattern and formation of nanofibers in capillary thinning of polymer solutions," Phys. Rev. Lett. 100, 164502 (2008).

[35] Sattler, R., S. Gier, J. Eggers, and C. Wagner, "The final stages of capillary break-up of polymer solutions," Phys. Fluids 24, 023101 (2012).

[36] Semakov, A. V., V. G. Kulichikhin, A. K. Tereshin, S. V. Antonov, and A. Y. Malkin, "On the nature of phase separation of polymer solutions at high extension rates," J. Polym. Sci. Pol. Phys. 53, 559-565 (2015).

[37] Malkin, A. Y., A. V. Semakov, I. Y. Skvortsov, P. Zatonskikh, V. G. Kulichikhin, A. V. Subbotin, and A. N. Semenov, "Spinnability of dilute polymer solutions," Macromolecules 50, 8231-8244 (2017).

[38] Deblais, A., K. P. Velikov, and D. Bonn, "Pearling instabilities of a viscoelastic thread," Phys. Rev. Lett. 120, 194501 (2018).

[39] Eggers, J., "Instability of a polymeric thread," Phys. Fluids 26, 033106 (2014).

[40] Helfand, E., and G. H. Fredrickson, "Large fluctuations in polymer solutions under shear," Phys. Rev. Lett. 62, 2468-2471 (1989).

[41] Doi, M., and A. Onuki, "Dynamic coupling between stress and composition in polymer solutions and blends," J. Phys. II France 2, 1631-1656 (1992).

[42] Milner, S. T., "Dynamical theory of concentration fluctuations in polymer solutions under shear," Phys. Rev. E 48, 3674-3691 (1993).

[43] Middleman, S., "Stability of a viscoelastic jet," Chem. Eng. Sci. 20, 1037-1040 (1965).

[44] Chang, H.-C., E. A. Demekhin, and E. Kalaidin, "Iterated stretching of viscoelastic jets," Phys. Fluids 11, 1717-1737 (1999).

[45] Li, J., and M. A. Fontelos, "Drop dynamics on the beads-on-string structure for viscoelastic jets: A numerical study," Phys. Fluids 15, 922-937 (2003).

[46] Bhat, P. P., S. Appathurai, M. T. Harris, M. Pasquali, G. H. McKinley, and O. A. Basaran, "Formation of beads-on-a-string structures during break-up of viscoelastic filaments," Nat. Phys. 6, 625-631 (2010).

[47] Bhat, P. P., O. A. Basaran, and M. Pasquali, "Dynamics of viscoelastic liquid filaments: Low capillary number flows," J. Nonnewton. Fluid Mech. 150, 211-225 (2008). 
[48] Subbotin, A. V., and A. N. Semenov, "Phase separation in dilute polymer solutions at high-rate extension,” J. Polym. Sci. Pol. Phys. 54, 1066-1073 (2016).

[49] Semenov, A. N., and A. V. Subbotin, "Phase separation kinetics in unentangled polymer solutions under high-rate extension," J. Polym. Sci. Pol. Phys. 55, 623-637 (2017).

[50] Subbotin, A. V., and A. N. Semenov, "Phase separation in polymer solutions under extension," Polym. Sci. C 60, S106-S117 (2018).

[51] Papkov, S. P., and V. G. Kulichikhin, The Liquid-Crystalline State of Polymers (Khimiya, Moscow, 1977).

[52] Semenov, A. N., and A. R. Khokhlov, "Statistical physics of liquidcrystalline polymers," Phys. Usp. 156, 988-1014 (1988).

[53] Larson, R. G., and D. W. Mead, "Linear viscoelasticity of nematic liquid crystalline polymers," J. Rheol. 33, 185-206 (1989).

[54] Rey, A. D., and M. M. Denn, "Dynamical phenomena in liquid-crystalline materials," Annu. Rev. Fluid Mech. 34, 233-266 (2002).

[55] Goyal, R. K., and M. M. Denn, "Orientational multiplicity and transitions in liquid crystalline droplets," Phys. Rev. E 75, 021704 (2007).

[56] Polk, M., T. L. Vigo, and A. F. Turbak, High performance fibers, in Encyclopedia of Polymer Science and Technology (John Wiley \& Sons, New York 2004), Vol. 10, pp. 198-230.

[57] Chae, H. G., and S. Kumar, "Rigid-Rod polymeric fibers," J. Appl. Polym. Sci. 100, 791-802 (2006).

[58] Afshari, M., D. J. Sikkema, K. Lee, and M. Bogle, "High performance fibers based on rigid and flexible polymers," Polym. Rev. 48, 230-274 (2008).

[59] Pignon, F., A. Magnin, J.-M. Piau, and G. G. Fuller, "The orientation dynamics of rigid rod suspensions under extensional flow," J. Rheol. 47, 371-388 (2003).

[60] Reneker, D. H., and I. Chun, "Nanometre diameter fibres of polymer, produced by electrospinning," Nanotechnology 7, 216-223 (1996).

[61] Fang, X., and D. H. Reneker, "DNA fibers by electrospinning," J. Macromol. Sci. B Phys. 36, 169-173 (1997).

[62] Wang, C., and T. Hashimoto, "Self-Organization in electrospun polymer solutions: From dissipative structures to ordered fiber structures through fluctuations," Macromolecules 51 , 4502-4515 (2018).

[63] Ma, A. W. K., F. Chinesta, T. Tuladhar, and M. R. Mackley, "Filament stretching of carbon nanotube suspensions," Rheol. Acta 47, 447-457 (2008).
[64] Tiwari, M. K., A. V. Bazilevsky, A. L. Yarin, and C. M. Megaridis, "Elongational and shear rheology of carbon nanotube suspensions," Rheol. Acta 48, 597-609 (2009).

[65] Darsono, N., H. Mizunuma, and H. Obara, "Extensional flow of carbon nanotube dispersion and its influence on electrical conductivity,” J. Soc. Rheol. Jpn. 40, 101-109 (2012).

[66] Doi, M., and S. F. Edwards, The Theory of Polymer Dynamics (Oxford University, New York, 1986).

[67] Teraoka, I., N. Ookubo, and R. Hayakawa, "Molecular theory on the entanglement effect of rodlike polymers," Phys. Rev. Lett. 55, 2712-2715 (1985).

[68] Landau, L. D., and E. M. Lifshitz, Statistical Physics (Pergamon, New York, 1980).

[69] McKinley, G. H., and A. Tripathi, "How to extract the Newtonian viscosity from capillary breakup measurements in a filament rheometer," J. Rheol. 44, 653-670 (2000).

[70] Maresov, E. A., and A. N. Semenov, "Mesoglobule morphologies of amphiphilic polymers," Macromolecules 41, 9439-9457 (2008).

[71] de Gennes, P. G., and J. Prost, The Physics of Liquid Crystals (Clarendon, Oxford, 1993).

[72] Martyushev, L. M., and V. D. Seleznev, "Maximum entropy production principle in physics, chemistry and biology," Phys. Rep. 426, 1-45 (2006).

[73] Lifshitz, I. M., and V. V. Slyozov, "The kinetics of precipitation from supersaturated solid solutions," J. Phys. Chem. Solids 19, 35-50 (1961).

[74] Gittes, F., B. Mickey, J. Nettleton, and J. Howard, "Flexural rigidity of microtubules and actin filaments measured from thermal fluctuations in shape," J. Cell Biol. 120, 923-934 (1993).

[75] Oosawa, F., and S. Asakura, Thermodynamics of the Polymerization of Protein (Academic, San Diego, 1975).

[76] van Mameren, J., K. C. Vermeulen, F. Gittes, and C. F. Schmidt, "Leveraging single protein polymers to measure flexural rigidity," J. Phys. Chem. B 113, 3837-3844 (2009).

[77] Moulin, E., F. Niess, M. Maaloum, E. Buhler, I. Nyrkova, and N. Giuseppone, "The hierarchical self-assembly of charge nanocarriers: A highly cooperative process promoted by visible light," Angew. Chem. Int. Ed. 49, 6974-6978 (2010).

[78] Nyrkova, I., E. Moulin, J. J. Armao IV, M. Maaloum, B. Heinrich, M. Rawiso, F. Niess, J.-J. Cid, N. Jouault, E. Buhler, A. N. Semenov, and N. Giuseppone, "Supramolecular self-assembly and radical kinetics in conducting self-replicating nanowires," ACS Nano 8 , 10111-10124 (2014). 